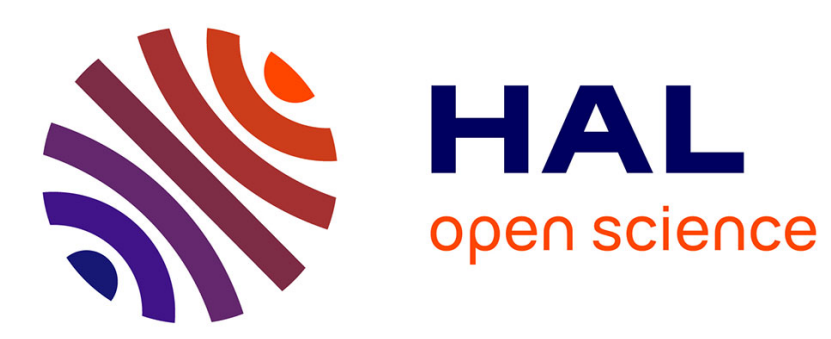

\title{
Some Rainbow Problems in Graphs Have Complexity Equivalent to Satisfiability Problems
}

Olivier Hudry, Antoine Lobstein

\section{To cite this version:}

Olivier Hudry, Antoine Lobstein. Some Rainbow Problems in Graphs Have Complexity Equivalent to Satisfiability Problems. International Transactions in Operational Research, 2022, 29 (3), pp.15471572. hal-02916921

\section{HAL Id: hal-02916921 \\ https://hal.science/hal-02916921}

Submitted on 30 Nov 2020

HAL is a multi-disciplinary open access archive for the deposit and dissemination of scientific research documents, whether they are published or not. The documents may come from teaching and research institutions in France or abroad, or from public or private research centers.
L'archive ouverte pluridisciplinaire HAL, est destinée au dépôt et à la diffusion de documents scientifiques de niveau recherche, publiés ou non, émanant des établissements d'enseignement et de recherche français ou étrangers, des laboratoires publics ou privés. 


\title{
Some Rainbow Problems in Graphs have Complexity Equivalent to Satisfiability Problems
}

\author{
Olivier Hudry \\ LTCI, Télécom Paris, Institut polytechnique de Paris \\ 19, place Marguerite Perey, 91120 Palaiseau, France \\ \& Antoine Lobstein \\ Centre National de la Recherche Scientifique \\ Laboratoire de Recherche en Informatique, UMR 8623, \\ Université Paris-Sud \\ Bâtiment 650 Ada Lovelace, 91405 Orsay Cedex - France \\ olivier.hudry@telecom-paris.fr, antoine.lobstein@lri.fr
}

June 29, 2020

\begin{abstract}
In a vertex-coloured graph, a set of vertices $S$ is said to be a rainbow set if every colour in the graph appears exactly once in $S$. We investigate the complexities of various problems dealing with domination in vertex-coloured graphs (existence of rainbow dominating sets, of rainbow locating-dominating sets, of rainbow identifying sets), including when we ask for a unique solution: we show equivalence between these complexities and those of the well-studied Boolean satisfiability problems.
\end{abstract}

Key Words: Graph theory, Complexity theory, Uniqueness of solution, Rainbow sets, Dominating codes, Locating-dominating codes, Identifying codes, Twin-free graphs 


\section{Introduction}

We intend to locate in the classes of complexity several problems linked to the existence of rainbow dominating sets, of rainbow locating-dominating sets, and of rainbow identifying sets in a vertex-coloured graph, including when we ask for a unique solution. To this effect, we shall prove equivalence (up to polynomials) between the complexities of our problems and those of satisfiability problems.

This work is motivated by [1], [2], in particular their Theorem 2.1 (see Proposition 10 below) on the complexity of finding rainbow dominating sets in a vertex-coloured graph. Since locating-dominating sets and identifying sets are particular classes of dominating sets, and since locatingdomination and identification are popular nowadays (see the ongoing bibliography at [24]), it seems natural to try to extend this theorem to these two classes, including when considering domination at distance $r$ and uniqueness of solution.

\subsection{Vertex-Coloured Graphs}

Let $G=(V, E)$ be a finite, simple, undirected graph with vertex set $V$ and edge set $E$, where an edge between $v_{1} \in V$ and $v_{2} \in V$ is indifferently denoted by $v_{1} v_{2}$ or $v_{2} v_{1}$. The order of $G$ is its number of vertices.

If $\Phi=\{1, \ldots, c\}$ is a set of colours, then $G_{c o l}=\left(V_{c o l}, E\right)$ denotes a vertex-coloured graph (or simply coloured graph) obtained from $G$ by giving one colour taken in $\Phi$ to every vertex $v \in V$, with each colour given to at least one vertex; here it is not necessary that two neighbour vertices receive different colours. When useful, we denote by $\phi(v)$ the colour given to $v$. A subset of vertices $V^{*} \subseteq V$ is said to be tropical if every colour appears at least once in $V^{*}$. It is said to be rainbow if every colour appears exactly once in $V^{*}$; therefore, any rainbow set has $c$ elements. When it is clear that we are dealing with a coloured graph, we shall often drop the subscript col.

Remark 1 Here, we stick to the definition of a rainbow subset which is used in [1] and [2], because our article is intended to prolong and widen the complexity result therein. However, a different terminology can be found in the literature: a rainbow subset can also designate a vertex subset where each colour appears at most once, whereas in a colourful subset, they appear exactly once; see, e.g., [3] or [9].

Since we shall study domination at distance $r$ and, to this purpose, use the term rainbow $r$-domination, we have to mention that $k$-rainbow domination is used with a different meaning in, e.g., [7].

\subsection{More Definitions and Notation in Graphs}

All the following definitions apply to graphs and coloured graphs. 
For any integer $r \geq 2$, the $r$-th power of $G$ is the graph $G^{r}=\left(V, E^{r}\right)$, with $E^{r}=\left\{v_{1} v_{2}: v_{1}, v_{2} \in V, 0<d_{G}\left(v_{1}, v_{2}\right) \leq r\right\}$.

For any integer $r \geq 1$, and for every vertex $v \in V$, we denote by $B_{G, r}(v)$ (and $B_{r}(v)$ when there is no ambiguity) the ball of radius $r$ centered at $v$, i.e., the set of vertices at distance at most $r$ from $v$ :

$$
B_{r}(v)=\left\{w \in V: 0 \leq d_{G}(v, w) \leq r\right\} .
$$

Whenever $v \in B_{r}(w)$ (which is equivalent to $w \in B_{r}(v)$ ), we say that $v$ and $w r$-dominate each other. When three vertices $v, w, z$ are such that $z \in B_{r}(v)$ and $z \notin B_{r}(w)$, we say that $z$-separates $v$ and $w$ in $G$ (note that $z=v$ is possible). A set of vertices is said to $r$-separate $v$ and $w$ if at least one of its elements does.

A subset of vertices $V^{*}$ will be indifferently called a set or a code, and its elements codewords. We denote by $I_{G, V^{*}, r}(v)$ (and $I_{r}(v)$ when there is no ambiguity) the set of codewords that $r$-dominate $v: I_{G, V^{*}, r}(v)=B_{G, r}(v) \cap$ $V^{*}$.

A code $V^{*}$ is said to be an $r$-dominating set or an $r$-dominating code $\left(r\right.$-D code for short) if for all $v \in V$, we have $I_{r}(v) \neq \emptyset$. One can also find the terminology dominating set at distance $r$, or distance $r$ dominating set.

A code $V^{*}$ is said to be $r$-locating-dominating ( $r$-LD for short) if for all $v \in V$, we have $I_{r}(v) \neq \emptyset$, and for any two distinct non-codewords $v_{1}, v_{2} \in V \backslash V^{*}$, we have $I_{r}\left(v_{1}\right) \neq I_{r}\left(v_{2}\right)$.

A code $V^{*}$ is said to be $r$-identifying ( $r$-ID for short) if for all $v \in V$, we have $I_{r}(v) \neq \emptyset$, and for any two distinct vertices $v_{1}, v_{2} \in V$, we have $I_{r}\left(v_{1}\right) \neq I_{r}\left(v_{2}\right)$.

In other words: every vertex must be $r$-dominated by at least one codeword for the three definitions; in addition, every pair of distinct noncodewords (respectively, vertices) must be $r$-separated by an $r$-LD (respectively, $r$-ID) code.

Two vertices $v_{1}, v_{2} \in V, v_{1} \neq v_{2}$, are said to be $r$-twins if $B_{r}\left(v_{1}\right)=$ $B_{r}\left(v_{2}\right)$. Dominating and locating-dominating codes exist for all graphs; on the other hand, it is easy to see that a graph $G$ admits an $r$-identifying code if and only if

$$
\forall v_{1} \in V, \forall v_{2} \in V, v_{1} \neq v_{2}: B_{r}\left(v_{1}\right) \neq B_{r}\left(v_{2}\right) .
$$

A graph satisfying (1) is called $r$-identifiable or $r$-twin-free. The following useful remarks are quite trivial and need no proofs.

Remark 2 Let $r \geq 2$ be any integer and $G=(V, E)$ be a graph.

(a) A code $V^{*}$ is 1-dominating in $G^{r}$, the $r$-th power of $G$, if and only if it is $r$-dominating in $G$.

(b) A code $V^{*}$ is 1-locating-dominating in $G^{r}$ if and only if it is $r$-locatingdominating in $G$.

(c) A code $V^{*}$ is 1-identifying in $G^{r}$ if and only if it is r-identifying in $G$. 
Remark $3 A$ code $V^{*}$ is $r$-ID (respectively, $r-L D$ ) if and only if (a) for every vertex $v \in V, I_{r}(v) \neq \emptyset$, and (b) for every pair of distinct vertices $v_{1}, v_{2} \in V$ (respectively, $v_{1}, v_{2} \in V \backslash V^{*}$ ), we have

$$
\left[B_{r}\left(v_{1}\right) \Delta B_{r}\left(v_{2}\right)\right] \cap V^{*} \neq \emptyset,
$$

where $\Delta$ stands for the symmetric difference.

For the vast topic of 1-domination in graphs, see [18]. For locating-dominating and identifying codes, see the large bibliography at [24].

\subsection{Satisfiability Problems}

We consider a set $\mathcal{X}$ of $n$ Boolean variables $x_{i}$ and a set $\mathcal{C}$ of $m$ clauses; each clause contains literals, a literal being a variable $x_{i}$ or its complement (or negated variable) $\bar{x}_{i}$. A truth assignment for $\mathcal{X}$ sets the variable $x_{i}$ to TRUE, also denoted by $\mathrm{T}$, and its complement to FALSE (or F), or vice-versa. A truth assignment is said to satisfy a clause if this clause contains at least one true literal, and to satisfy the set of clauses $\mathcal{C}$ if every clause contains at least one true literal. The following decision problems, for which the size of the instance is polynomially linked to $n+m$, are classical problems in complexity.

Problem SAT (Satisfiability):

Instance: A set $\mathcal{X}$ of variables, a collection $\mathcal{C}$ of clauses over $\mathcal{X}$, each clause containing at least two different literals.

Question: Is there a truth assignment for $\mathcal{X}$ that satisfies $\mathcal{C}$ ?

Problem 3-SAT (3-Satisfiability):

Instance: A set $\mathcal{X}$ of variables, a collection $\mathcal{C}$ of clauses over $\mathcal{X}$, each clause containing exactly three different literals.

Question: Is there a truth assignment for $\mathcal{X}$ that satisfies $\mathcal{C}$ ?

\subsection{A Short Background on Complexity}

See, e.g., [4], [16], [23] or [26] for more on this topic; we assume that the reader is already familiar with the classes $P, N P$ and co- $N P$, with $N P$ complete problems, and with the notion of polynomial transformation between problems.

For problems which are not necessarily decision problems, a Turing reduction from a problem $\pi_{1}$ to a problem $\pi_{2}$ is an algorithm $\mathcal{A}$ that solves $\pi_{1}$ using a (hypothetical) subprogram $\mathcal{S}$ solving $\pi_{2}$ such that, if $\mathcal{S}$ were a polynomial algorithm for $\pi_{2}$, then $\mathcal{A}$ would be a polynomial algorithm for $\pi_{1}$. Thus, in this sense, $\pi_{2}$ is "at least as hard" as $\pi_{1}$. A problem $\pi$ is $N P$ hard (respectively, co-NP-hard) if there is a Turing reduction from some $N P$-complete (respectively, co- $N P$-complete) problem to $\pi[16, \mathrm{p} .113]$. 


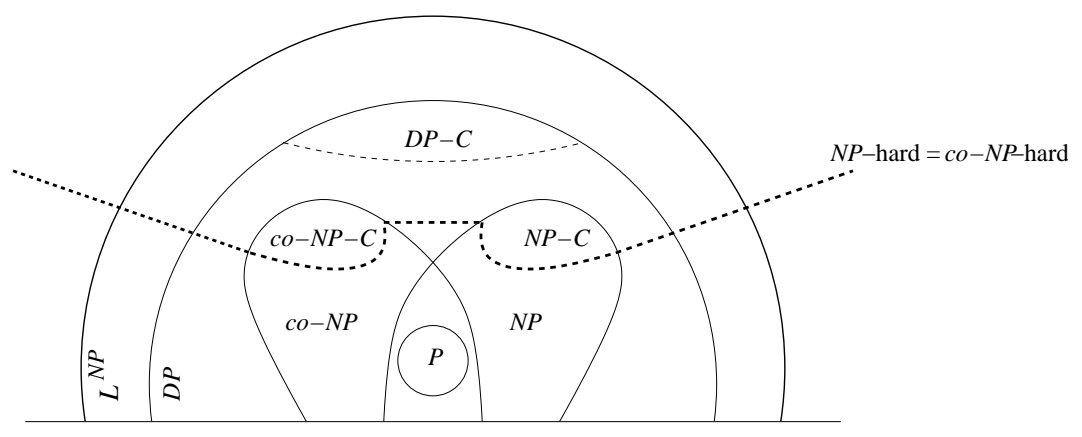

Figure 1: Some classes of complexity.

Remark 4 Note that these two definitions, NP-hard and co-NP-hard, coincide [16, p. 114].

The notions of completeness and hardness can be extended to classes other than $N P$ or co- $N P$.

We shall also use the class $L^{N P}[23]$ (also denoted by $P^{N P[O(\log n)]}$ or $\Theta_{2}$ ), which contains the decision problems which can be solved by applying, with a number of calls which is logarithmic with respect to the size of the instance, a subprogram able to solve an appropriate problem in $N P$ (usually, an $N P$ complete problem); and the class $D P$ [27] (or $D I F^{P}$ [5] or $B H_{2}$ [23], [28]) as the class of languages (or problems) $\mathcal{L}$ such that there are two languages $\mathcal{L}_{1} \in N P$ and $\mathcal{L}_{2} \in$ co- $N P$ satisfying $\mathcal{L}=\mathcal{L}_{1} \cap \mathcal{L}_{2}$. This class is not to be confused with $N P \cap$ co- $N P$ (see the warning in, e.g., [26, p. 412]); actually, $D P$ contains $N P \cup$ co- $N P$ and is contained in $L^{N P}$. See Figure 1.

Membership to $P, N P$, co- $N P, D P$ or $L^{N P}$ gives an upper bound on the complexity of a problem (this problem is not more difficult than ...), whereas a hardness result gives a lower bound (this problem is at least as difficult as ...). Still, such results are conditional in some sense; if for example $P=N P$, they would lose their interest. But we do not know whether or where the classes of complexity collapse.

The decision problems SAT and 3-SAT are two of the basic and most well-known $N P$-complete problems [14], [16, p. 39, p. 46 and p. 259]. If we consider their variants U-SAT and U-3-SAT, where the question now is "Is there a unique assignment ...", the following result was proved in [22].

Proposition 5 [22, Th. 10] The decision problems U-SAT and U-3-SAT have equivalent complexities, up to polynomials.

Using results from [5] and [26, p. 415], it is then rather simple to obtain the following result.

Corollary 6 (a) The decision problems U-SAT and U-3-SAT are NP-hard. (b) The decision problems U-SAT and U-3-SAT belong to the class DP. 
Remark 7 It is not known whether these problems are DP-complete. In [26, p. 415], it is said that "U-SAT is not believed to be DP-complete". It is shown in [5] that there exists one oracle under which U-SAT is not DPcomplete; and one oracle under which it is, if $N P \neq c o-N P$.

Note that uniqueness of solutions, which may be seen as part of the wider and rather unexplored issue of the number of solutions of a problem, had been studied earlier in a few papers (see, e.g., [5], [6], [8], [15], [17], [25]).

Let us now turn to the decision problems arising from the definitions given in Section 1.2; they are all stated for a fixed integer $r \geq 1$ :

Problem $\mathrm{DC}_{r} / \mathrm{LDC}_{r} / \mathrm{IDC}_{r}$ (\{r-Dominating / $r$-Locating-Dominating / $r$-IDentifying\} Code with bounded size):

Instance: A graph $G$ and an integer $k$.

Question: Does $G$ admit an $\{r$-dominating / $r$-locating-dominating $/ r$ identifying $\}$ code of size at most $k$ ?

Proposition 8 Let $r \geq 1$ be any integer.

(a) [16, p. 75 and $p .190$, for $r=1]$, [19, Prop. 9] The decision problem $D C_{r}$ is NP-complete.

(b) $[13$, for $r=1],[10]$ The decision problem $L D C_{r}$ is NP-complete.

(c) $[12$, for $r=1],[10]$ The decision problem IDC $C_{r}$ is NP-complete.

We have also results on the complexities of these problems when the question is about the uniqueness of the existence of a suitable set:

Proposition 9 Let $r \geq 1$ be any integer.

(a) [21, Th. 25] The decision problems $U-S A T$ and $U-D C_{r}$ have equivalent complexities, up to polynomials.

(b) [20, Th. 20] The decision problems U-SAT and $U-L D C_{r}$ have equivalent complexities, up to polynomials.

(c) [20, Th. 35] The decision problems U-SAT and U-IDC $C_{r}$ have equivalent complexities, up to polynomials.

As a consequence, by Corollary 6 , the problems $\mathrm{U}_{-} \mathrm{DC}_{r}, \mathrm{U}-\mathrm{LDC}_{r}$ and U$\mathrm{IDC}_{r}$ are $N P$-hard and belong to $D P$.

What about the same problems (with or without uniqueness of solution) when we consider coloured graphs? Note that it would not be interesting to ask whether there is, e.g., a tropical $r$-dominating code of size at most $k$ in a coloured graph $G_{c o l}$ : in this case, we can simply observe that with a graph coloured with only one colour, we are brought back to the basic problem $\mathrm{DC}_{r}$. Much more interesting is to consider the existence of rainbow sets, that is, to try to locate the following problems in the classes of complexity, and this is what we shall do in the sequel, with the additional requirement that all graphs be connected (see Remark 23): 
Problem [U-] $\left\{\mathrm{RDC}_{r} / \mathrm{RLDC}_{r} / \mathrm{RIDC}_{r}\right\}$ ([Unique] Rainbow $\{r$-Dominating $/ r$-Locating-Dominating / $r$-IDentifying $\}$ Code):

Instance: A connected, coloured graph $G_{c o l}$.

Question: Does $G_{c o l}$ admit a [unique] rainbow $\{r$-dominating / $r$-locatingdominating / $r$-identifying\} code?

\subsection{Outline of the Paper}

In Sections 2.1-2.3, we give results on the $N P$-completeness of the problems $\mathrm{RDC}_{1}, \mathrm{RLDC}_{1}$ and $\mathrm{RIDC}_{1}$.

In Sections 2.4-2.6, we prove that the three problems $\mathrm{U}-\mathrm{RDC}_{1}$, U$\mathrm{RLDC}_{1}$ and $\mathrm{U}_{-\mathrm{RIDC}}$ have a complexity which is equivalent to that of U-SAT or U-3-SAT.

In some cases, we have results which hold even for paths or trees, or for graphs with a small number of occurrences for the colours appearing in the graph (typically, 2 or 3 ).

Then, in Sections 2.7-2.9, we shall extend our results to any $r$.

For the three types of codes, the general approach is the same, but the results slightly vary, and each type requires proofs which are different in their technical details, and cannot be merged. The starting point is the following result.

Proposition 10 [1, Th. 2.1], [2, Th. 2.1] The problem $R D C_{1}$ is NPcomplete, even when restricted to coloured paths.

We give the proof, because it will be used and transformed for subsequent proofs. When, during the construction of a coloured graph, we say that a vertex has or is given a unique colour, we mean that this colour, at the end of the construction, appears exactly once. By extension, a vertex with unique colour is said to be unique, so that any unique vertex necessarily belongs to any rainbow set.

Proof of Proposition 10. In view of the subsequent proofs, we change slightly the proof from [1], [2]. The problem is clearly inside $N P$. We give a polynomial transformation from 3 -SAT to $\mathrm{RDC}_{1}$. Let the set $\mathcal{C}$ of $m$ clauses over $n$ variables $x_{1}, x_{2}, \ldots, x_{n}$ be an instance of 3-SAT, for which we may assume that each variable $x_{i}$ appears with its two forms, $x_{i}$ and $\bar{x}_{i}$ (otherwise, if it appears only in, say, its negated form, it suffices to take $x_{i}=\mathrm{F}$, and this variable and the clauses where it appears do not need to be considered anymore).

We write $\mathcal{C}=\left\{\left\{\ell_{1}, \ell_{2}, \ell_{3}\right\},\left\{\ell_{4}, \ell_{5}, \ell_{6}\right\}, \ldots,\left\{\ell_{3 m-2}, \ell_{3 m-1}, \ell_{3 m}\right\}\right\}$, where each literal $\ell_{j}$ is a variable $x_{i}$ or its complement $\bar{x}_{i}$. From this instance, we define a coloured path $\mathcal{P}$ such that $\mathcal{P}$ admits a rainbow 1-dominating set if and only if there is an assignment of the variables that satisfies $\mathcal{C}$. Example 12 below is intended to help to understand the notation. 


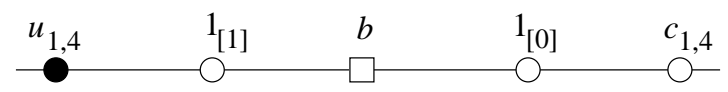

artificial $\mathrm{A}$ positive $\mathrm{P}$ middle $\mathrm{M}$ negative $\mathrm{N}$ link $\mathrm{L}$

Figure 2: The gadget $W_{1,4}(i=1, i(1)=4)$. The artificial vertex is represented by a black circle because it belongs to any rainbow set, the middle vertex by a square because it does not belong to any rainbow 1-dominating set, the other three vertices are unspecified.

We first construct a path

$$
\mathcal{P}_{0}=z_{1} z_{2} S_{1} v_{1} v_{2} v_{3} S_{2} v_{4} v_{5} v_{6} S_{3} \ldots S_{m} v_{3 m-2} v_{3 m-1} v_{3 m} S_{m+1},
$$

and we colour it in the following way. Vertices $z_{1}$ and $z_{2}$ are Blue (colour $b$ ). Each vertex $S_{s}, 1 \leq s \leq m+1$, receives a unique colour. Each vertex $v_{i}$, $1 \leq i \leq 3 m$, which corresponds to the literal $\ell_{i}$ and is called a clausal vertex, is coloured with the colour $\phi\left(v_{i}\right)=i_{[0]}$.

Next, we define a number of gadgets as follows. Whenever a pair of literals $\ell_{p}, \ell_{q}$ satisfies $\ell_{p}=\bar{\ell}_{q}$, we say that they are antithetic to each other, and the same applies for the corresponding clausal vertices $v_{p}$ and $v_{q}$. For each literal $\ell_{i}, 1 \leq i \leq 3 m$, we consider the list of all the literals $\ell_{i(1)}, \ell_{i(2)}, \ldots, \ell_{i\left(k_{i}\right)}$, that are antithetic to $\ell_{i}$ (by assumption, there is at least one). To each literal $\ell_{i(f)}, 1 \leq f \leq k_{i}$, is associated a constraint gadget $W_{i, i(f)}$ consisting of a path on five vertices, $A_{i, i(f)}, P_{i, i(f)}, M_{i, i(f)}, N_{i, i(f)}$ and $L_{i, i(f)}$; vertex $A_{i, i(f)}$ is called artificial and has a unique colour, $u_{i, i(f)}$; vertex $P_{i, i(f)}$ is the positive vertex of $W_{i, i(f)}$ and has colour $i_{[f]}$; vertex $M_{i, i(f)}$ is the middle vertex of $W_{i, i(f)}$, and $\phi\left(M_{i, i(f)}\right)=b$; vertex $N_{i, i(f)}$ is the negative vertex of $W_{i, i(f)}$ and has colour $i_{[f-1]}$; vertex $L_{i, i(f)}$ is the link vertex of $W_{i, i(f)}$ and has colour $c_{i, i(f)}$ if $i<i(f)$, colour $c_{i(f), i}$ otherwise.

Remark 11 (a) In $\mathcal{P}$, the vertex $z_{1}$ will not be linked to any vertex other than $z_{2}$; therefore, $z_{1}$ or $z_{2}$ necessarily belongs to any 1-dominating set in $\mathcal{P}$, and no Blue vertex other than $z_{1}$ or $z_{2}$ can belong to any rainbow 1-dominating set in $\mathcal{P}$, i.e., no middle vertex.

(b) Every vertex $S_{s}, 1 \leq s \leq m+1$, and every artificial vertex $A_{i, i(f)}$, $1 \leq i \leq 3 m, 1 \leq f \leq k_{i}$, necessarily belong to any rainbow set.

(c) Every positive vertex $P_{i, i\left(k_{i}\right)}$ (which has colour $i_{\left[k_{i}\right]}$ ) is unique (see Remark 13 below) and belongs to any rainbow set.

Example 12 Assume that $\ell_{1}=\ell_{7}=\ell_{13}=x_{1}, \ell_{4}=\ell_{10}=\bar{x}_{1}$ and there is no other occurrence of $x_{1}$ nor $\bar{x}_{1}$ in $\mathcal{C}$. Figure 2 represents the gadget $W_{1,4}$.

We have $k_{1}=k_{7}=k_{13}=2, k_{4}=k_{10}=3$, and $\ell_{1(1)}=\ell_{4}, \ell_{1(2)}=\ell_{10}$, $\ell_{7(1)}=\ell_{4}, \ldots, \ell_{10(3)}=\ell_{13}$. 


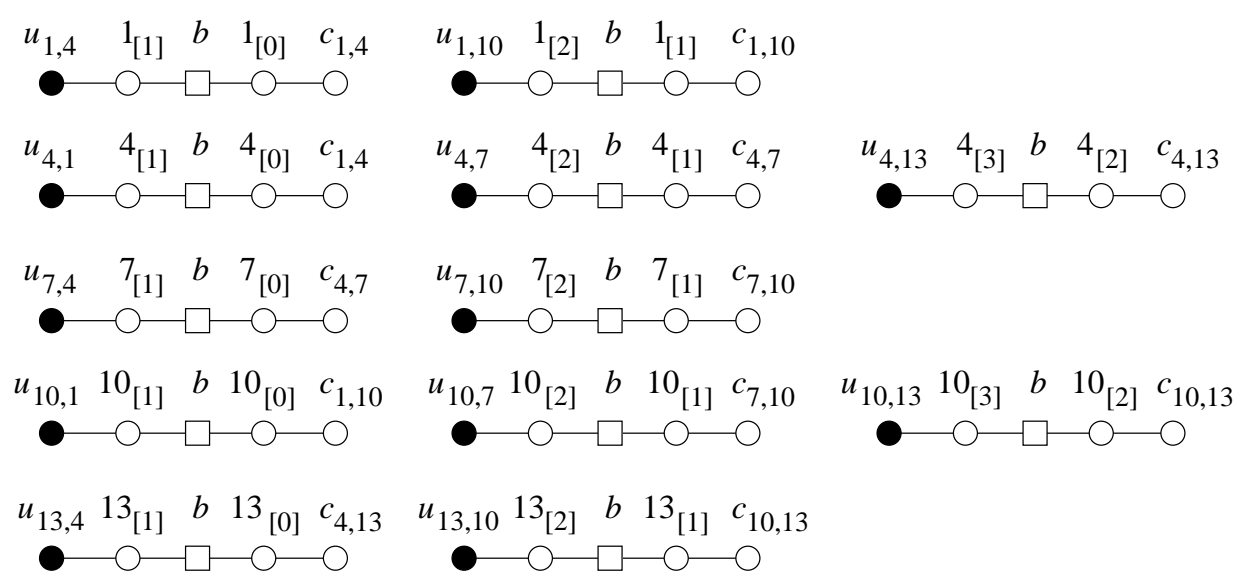

Figure 3: The 12 gadgets from Example 12.

Figure 3 represents the 12 gadgets $W_{1,4}, W_{1,10}, W_{4,1}, W_{4,7}, W_{4,13}, W_{7,4}$, $W_{7,10}, W_{10,1}, W_{10,7}, W_{10,13}, W_{13,4}, W_{13,10}$, produced by $\ell_{1}=x_{1}, \ell_{4}=\bar{x}_{1}$, $\ell_{7}=x_{1}, \ell_{10}=\bar{x}_{1}, \ell_{13}=x_{1}$.

Remark 13 One can see that the colours $c_{i, i(f)}$ appear exactly twice, on the link vertices of the gadgets $W_{i, i(f)}$ and $W_{i(f), i}$, and the same is true for the colours $i_{[0]}$ (on one clausal vertex and in one gadget), $i_{[1]}$ (in two gadgets), ..., except for the "last" colour $i_{\left[k_{i}\right]}$, which appears only once, on the positive vertex $P_{i, i\left(k_{i}\right)}$ (in Figure 3 , these colours are $1_{[2]}, 4_{[3]}, 7_{[2]}, 10_{[3]}$ and $13_{[2]}$ ). Only the Blue colour appears more than twice.

Finally, the path $\mathcal{P}$ is obtained by concatenating $\mathcal{P}_{0}$ and all the different gadgets, and creating a unique vertex $J$, which is linked to the last vertex of the last gadget. The gadgets are ordered lexicographically; thus, in our example, we obtain $\mathcal{P}=\mathcal{P}_{0} W_{1,4} W_{1,10} W_{4,1} \ldots W_{13,10} J$.

Clearly, the construction is polynomial in the size of the instance of 3-SAT.

(a) We assume that there is a solution to 3-SAT, i.e., an assignment of the variables that satisfies $\mathcal{C}$. We construct a rainbow 1-dominating set $V^{*}$ by putting the following vertices in $V^{*}$ : (i) the vertex $z_{1}$ and all the unique vertices; (ii) for every true literal $\ell_{i}$, the clausal vertices $v_{i}$ (with colour $i_{[0]}$ ) and, for every $f \in\left\{1, \ldots, k_{i}\right\}$, the positive vertices $P_{i, i(f)} \in W_{i, i(f)}$ and the link vertices $L_{i, i(f)} \in W_{i, i(f)}$; (iii) for every false literal $\ell_{i}$ and for every $f \in\left\{1, \ldots, k_{i}\right\}$, the negative vertices $N_{i, i(f)} \in W_{i, i(f)}$.

See Figure 4 for the gadgets of Example 12 with $x_{1}=$ FALSE. Note that some vertices may be codewords for two reasons, (i) and (ii). In Figure 4, this is the case for the two positive vertices with colours $4_{[3]}$ and $10_{[3]}$ (the two large black vertices). 


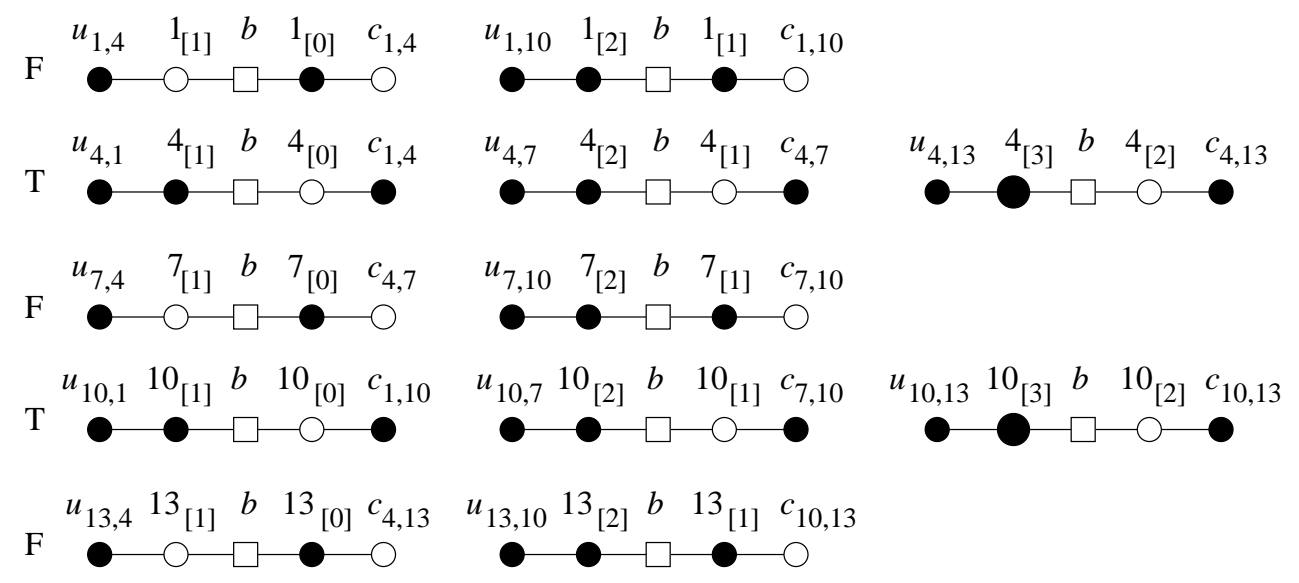

Figure 4: In black, the codewords in the 12 gadgets from Example 12, with $x_{1}=\mathrm{F}$, i.e., $\ell_{1}=\mathrm{F}, \ell_{4}=\mathrm{T}, \ell_{7}=\mathrm{F}, \ell_{10}=\mathrm{T}, \ell_{13}=\mathrm{F}$.

We claim that $V^{*}$ is both rainbow and 1-dominating.

It is straightforward to check that the Blue colour, the unique colours and every colour $i_{[f]}, 1 \leq i \leq 3 m, 0 \leq f \leq k_{i}$, appear exactly once in $V^{*}$. All that remains to be checked are the colours $c_{i, i(f)}$ of the link vertices in the gadgets $W_{i, i(f)}$ and $W_{i(f), i}$; since these two gadgets correspond to two literals that are antithetic, exactly one of them has been set TRUE by the assignment and exactly one of these two link vertices has been taken in the code. So $V^{*}$ is rainbow.

For every $s \in\{1, \ldots, m\}$, the clausal vertices $v_{3 s-2}$ and $v_{3 s}$ are 1dominated by $S_{s} \in V^{*}$ and $S_{s+1} \in V^{*}$, respectively; since by assumption there is at least one true literal in each clause, the clausal vertex $v_{3 s-1}$ is 1-dominated by at least one clausal vertex which is a codeword. Inside a gadget, either the negative vertex, or the positive and link vertices, are codewords, and in both cases, every vertex is 1-dominated by $V^{*}$. Hence $V^{*}$ is a 1-dominating set.

(b) We assume that there is a solution to $\mathrm{RDC}_{1}$, i.e., a rainbow 1dominating set $V^{*}$. By Remark 11(a), no middle vertex can belong to $V^{*}$.

For every clausal vertex $v_{i}$ (with colour $i_{[0]}$ ) that is a codeword, we assign the value to the corresponding variable in such a way that $\ell_{i}$ is TRUE. We claim that this (partial) assignment is consistent. Assume on the contrary that there is a pair of antithetic literals $\ell_{p}$ and $\ell_{q}$ receiving the same value by the assignment just defined, i.e., that the two antithetic clausal vertices $v_{p}$ and $v_{q}$ are codewords. Assume without loss of generality that $p<q$. There is an $f \geq 1$ such that $\ell_{q}=\ell_{p(f)}$, and two gadgets, $W_{p, p(f)}$ and $W_{p(f), p}$, both containing a link vertex with the common colour $c_{p, p(f)}$.

Consider $W_{p, p(f)}$ and its link vertex $L_{p, p(f)}$, and assume that $L_{p, p(f)} \notin$ 
$V^{*}$. Assume first that $f>1$. Because $M_{p, p(f)} \notin V^{*}$, we have $N_{p, p(f)} \in$ $V^{*}$, then $P_{p, p(f-1)} \notin V^{*}$ (because it has the same colour as $\left.N_{p, p(f)}\right)$, then $N_{p, p(f-1)} \in V^{*}$ (to have one codeword that 1-dominates $\left.M_{p, p(f-1)}\right), \ldots$, $N_{p, p(1)} \in V^{*}$; but $N_{p, p(1)}$ has colour $p_{[0]}$, like $v_{p}$ which is also a codeword, and this contradicts the fact that $V^{*}$ is rainbow. If $f=1$, we get immediately the same conclusion. So $L_{p, p(f)}$ must belong to $V^{*}$. But the same argument can be applied to $W_{p(f), p}$ and $L_{p(f), p}$, so the two link vertices, which have the same colour, are codewords, which is impossible. Therefore, the assignment defined by $V^{*}$ is valid. If necessary, we complete the assignment by giving the value TRUE to the remaining unassigned variables.

Finally, this assignment satisfies 3-SAT because, for domination reasons, in each clause at least one clausal vertex belongs to $V^{*}$, i.e., at least one literal is true.

So the answer to the initial instance of 3-SAT is YES if and only if the answer to the constructed instance of $\mathrm{RDC}_{1}$ is $\mathrm{YES}$.

\section{New Results}

The first three Subsections of this Section are devoted to the problems $\mathrm{RDC}_{1}, \mathrm{RLDC}_{1}$ and $\mathrm{RIDC}_{1}$ : we study one variant for $\mathrm{RDC}_{1}$, then we prove that $\mathrm{RLDC}_{1}$ and $\mathrm{RIDC}_{1}$ are $N P$-complete, i.e., their complexity is equivalent, up to polynomials, to that of, e.g., SAT.

The following three Subsections show that the same three problems with unique solution have complexity equivalent, up to polynomials, to that of U-SAT.

Then, in Subsections 2.7-2.9, we shall extend our results to $\mathrm{RDC}_{r}$, $\mathrm{RLDC}_{r}$ and $\mathrm{RIDC}_{r}$, for any $r>1$.

\subsection{Rainbow 1-Dominating Codes}

We can go further and ask for a fixed number of occurrences for the colours appearing in the graph. With this number restricted to 2 , we have a result of NP-completeness for coloured trees.

Proposition 14 The problem $R D C_{1}$ is NP-complete, even when restricted to trees where each colour appears at most twice.

Proof. We consider the construction in the proof of Proposition 10, where every middle vertex $M_{i, i(f)}$ in the gadget $W_{i, i(f)}$ had been given the colour $b$. As noticed in Remark 13, the Blue colour is the only colour appearing more than twice in the graph. To get rid of these multiple occurrences, we proceed as follows: (i) we delete the vertices $z_{1}, z_{2}$ and the edges $z_{1} z_{2}$ and $z_{2} S_{1}$; (ii) for every middle vertex, we set $\phi\left(M_{i, i(f)}\right)=b_{i, i(f)}$ and we create the graph $H_{i, i(f)}=\left(V_{i, i(f)}, E_{i, i(f)}\right)$ with 
(a)

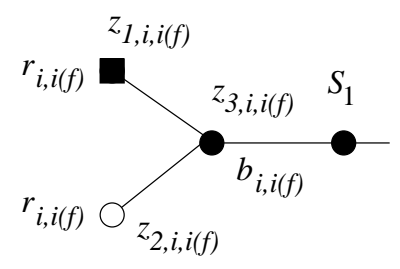

(b)

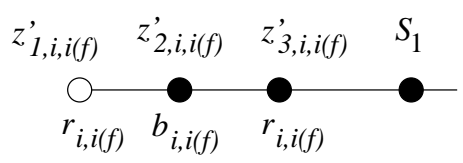

Figure 5: (a) The graph $H_{i, i(f)}$ of Propositions 14 and 17, with its link to $S_{1}$; (b) The graph $H_{i, i(f)}^{\prime}$ of Propositions 19 and 28, with its link to $S_{1}$. In black, the codewords, in white the non-codewords; black circles are forced codewords.

(a)

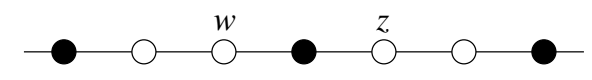

(b1)

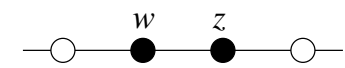

(b2)

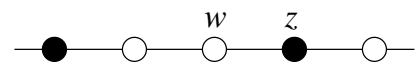

Figure 6: In black, codewords, and in white, non-codewords. The vertices $w$ and $z$ are not 1 -separated by any codeword.

$$
V_{i, i(f)}=\left\{z_{1, i, i(f)}, z_{2, i, i(f)}, z_{3, i, i(f)}\right\}, E_{i, i(f)}=\left\{z_{1, i, i(f)} z_{3, i, i(f)}, z_{2, i, i(f)} z_{3, i, i(f)}\right\},
$$

together with the edge $z_{3, i, i(f)} S_{1}$. We set $\phi\left(z_{3, i, i(f)}\right)=b_{i, i(f)}$ and give a new colour, say $r_{i, i(f)}$ (for Red), to both $z_{1, i, i(f)}$ and $z_{2, i, i(f)}$ : see Figure $5(\mathrm{a})$. Now the new graph is a tree and has no colour appearing more than twice. Since the Red vertices $z_{1, i, i(f)}$ and $z_{2, i, i(f)}$ must be 1-dominated by some codeword and cannot both belong to a rainbow set, we see that $z_{3, i, i(f)}$ necessarily belongs to any rainbow 1-dominating code (together with exactly one of $z_{1, i, i(f)}$ and $\left.z_{2, i, i(f)}\right)$; the consequence is that again, no middle vertex can belong to any rainbow 1-dominating set. The proof then goes exactly as for Proposition 10.

\subsection{Rainbow 1-Locating-Dominating Codes}

We now turn to 1-locating-dominating codes. We obtain the same results as for 1-dominating codes, one for paths without limitation on the occurrences of colours, one for trees where every colour appears at most twice.

Remark 15 On a path, the only configuration for a set which is 1-dominating and not 1-LD is given by Figure 6(a). One can see that this will never occur in the gadgets described in the proof of Proposition 10.

Proposition 16 The problem $R L D C_{1}$ is NP-complete, even when restricted to coloured paths. 
Proof. We consider again the construction in the proof of Proposition 10. We duplicate the vertices $S_{s}, 1 \leq s \leq m$, so the path $\mathcal{P}_{0}$ now reads

$$
\mathcal{P}_{0}=z_{1} z_{2} S_{1} S_{1}^{\prime} v_{1} v_{2} v_{3} S_{2} S_{2}^{\prime} v_{4} v_{5} v_{6} S_{3} S_{3}^{\prime} \ldots S_{m} S_{m}^{\prime} v_{3 m-2} v_{3 m-1} v_{3 m} S_{m+1},
$$

where each $S_{s}^{\prime}$ is unique. The proof now goes exactly as previously:

(a) We construct a code $V^{*}$ by following the same rules (i)-(iii) as in the Case (a) of the proof of Proposition 10. It is straightforward to see that all non-codewords are 1-dominated and 1-separated by the code in the gadgets (cf. Remark 15) as well as in $\mathcal{P}_{0}$, since the duplications avoid the configurations of the type:

$v_{3 s-2} \in V^{*}, v_{3 s-1} \notin V^{*}, v_{3 s} \notin V^{*}, S_{s+1} \in V^{*}, v_{3 s+1} \notin V^{*}, v_{3 s+2} \notin V^{*}$, $v_{3 s+3} \in V^{*}$

(cf. Figure 6(a)), that could exist in the previous construction. So $V^{*}$ is a rainbow 1-LD set.

(b) Assume that there exists a rainbow 1-LD set $V^{*}$. Then we can apply mutatis mutandis the Case (b) of the proof of Proposition 10, and construct a valid assignment satisfying 3-SAT.

Proposition 17 The problem $R L D C_{1}$ is NP-complete, even when restricted to trees where each colour appears at most twice.

Proof. Compared to the proof of Proposition 10, we duplicate the vertices $S_{s}, 1 \leq s \leq m$, like we did for Proposition 16, and, like for Proposition 14, (i) we delete the vertices $z_{1}, z_{2}$ and the edges $z_{1} z_{2}$ and $z_{2} S_{1}$; (ii) for every middle vertex, we set $\phi\left(M_{i, i(f)}\right)=b_{i, i(f)}$ and we create the same graph $H_{i, i(f)}$ and the edge $z_{3, i, i(f)} S_{1}$, cf. Figure 5(a). Again, the new graph is a tree and has no colour appearing more than twice, and $z_{3, i, i(f)}$ necessarily belongs to any rainbow $1-\mathrm{LD}$ code. The proof then goes like the previous ones.

\subsection{Rainbow 1-Identifying Codes}

We now consider 1-identifying codes. We do not obtain a result which would be valid for paths, as was the case for the problems $\mathrm{RDC}_{1}$ and $\mathrm{RLDC}_{1}$, but we do have a result on trees with occurrences of colours at most 2 .

Remark 18 On a path, the only three configurations for a set which is dominating and not ID are given by Figure 6(a)-(b1)-(b2).

Proposition 19 The problem $R I D C_{1}$ is $N P$-complete, even when restricted to trees where each colour appears at most twice.

Proof. We consider again the construction for the proof of Proposition 10 .

(i) We triplicate the vertices $S_{s}, 1 \leq s \leq m+1$, so the path $\mathcal{P}_{0}$ now reads

$$
z_{1} z_{2} S_{1} S_{1}^{\prime} S_{1}^{\prime \prime} v_{1} v_{2} v_{3} S_{2} S_{2}^{\prime} S_{2}^{\prime \prime} v_{4} \ldots S_{m} S_{m}^{\prime} S_{m}^{\prime \prime} v_{3 m-2} v_{3 m-1} v_{3 m} S_{m+1} S_{m+1}^{\prime} S_{m+1}^{\prime \prime},
$$


where $S_{s}^{\prime}$ and $S_{s}^{\prime \prime}$ are unique. We also triplicate all the artificial vertices, and the gadget $W_{i, i(f)}$ now reads $A_{i, i(f)} A_{i, i(f)}^{\prime} A_{i, i(f)}^{\prime \prime} P_{i, i(f)} M_{i, i(f)} N_{i, i(f)} L_{i, i(f)}$, where $A_{i, i(f)}^{\prime}$ and $A_{i, i(f)}^{\prime \prime}$ are unique. Moreover, we triplicate the final vertex $J$ by creating the unique vertices $J^{\prime}$ and $J^{\prime \prime}$, together with the edges $J J^{\prime}$ and $J^{\prime} J^{\prime \prime}$.

(ii) For each middle vertex $M_{i, i(f)} \in W_{i, i(f)}$, we create the unique vertex $Y_{i, i(f)}$, which is linked to $M_{i, i(f)}$ : this will avoid the configuration given by Figure 6(b2), which appears for instance in the first gadget of Figure 4. The graph thus constructed is already not a path anymore.

(iii) We get rid of the multiple Blue colours of the middle vertices by deleting the vertices $z_{1}, z_{2}$ and the edges $z_{1} z_{2}$ and $z_{2} S_{1}$, and creating, for every middle vertex $M_{i, i(f)}$, the graph $H_{i, i(f)}^{\prime}=\left(V_{i, i(f)}^{\prime}, E_{i, i(f)}^{\prime}\right)$, with

$$
V_{i, i(f)}^{\prime}=\left\{z_{1, i, i(f)}^{\prime}, z_{2, i, i(f)}^{\prime}, z_{3, i, i(f)}^{\prime}\right\}, E_{i, i(f)}^{\prime}=\left\{z_{1, i, i(f)}^{\prime} z_{2, i, i(f)}^{\prime}, z_{2, i, i(f)}^{\prime} z_{3, i, i(f)}^{\prime}\right\},
$$

together with the edge $z_{3, i, i(f)}^{\prime} S_{1}$. The colours are: $\phi\left(M_{i, i(f)}\right)=\phi\left(z_{2, i, i(f)}^{\prime}\right)=$ $b_{i, i(f)}$ and $\phi\left(z_{1, i, i(f)}^{\prime}\right)=\phi\left(z_{3, i, i(f)}^{\prime}\right)=r_{i, i(f)}$, see Figure $5(\mathrm{~b})$. The only vertex 1-separating $z_{1, i, i(f)}^{\prime}$ and $z_{2, i, i(f)}^{\prime}$ is $z_{3, i, i(f)}^{\prime}$, so $z_{3, i, i(f)}^{\prime}$ belongs to every 1-identifying code. Then $z_{1, i, i(f)}^{\prime}$ cannot be a codeword, and $z_{2, i, i(f)}^{\prime}$ is a codeword: observe already that here, we have no choice for these three vertices, unlike in the case of the graphs $H_{i, i(f)}$ for rainbow 1-D and 1-LD codes.

The graph $G$ just constructed is a tree, and no colour appears more than twice.

(a) We construct a code $V^{*}$ in $G$ by following the same rules (i)-(iii) as in the Case (a) of the proof of Proposition 10. In particular, every artificial vertex and every $Y_{i, i(f)}$ are codewords, because they are unique, and inside a gadget, either the negative vertex, or the positive and link vertices, are codewords. It is easy to see why the three forbidden configurations of Figure 6 cannot appear in $\mathcal{P}_{0}$. Also thanks to the triplications, inside each gadget, the artificial, positive and link vertices are all 1-dominated and 1-separated from all vertices by the artificial vertices.

If we have taken $N_{i, i(f)}$ in the code, then $I_{G, V^{*}, 1}\left(N_{i, i(f)}\right)=\left\{N_{i, i(f)}\right\}$ and $I_{G, V^{*}, 1}\left(M_{i, i(f)}\right)=\left\{N_{i, i(f)}, Y_{i, i(f)}\right\}$.

If we have taken $P_{i, i(f)}$ and $L_{i, i(f)}$ in the code, then $I_{G, V^{*}, 1}\left(N_{i, i(f)}\right)=$ $\left\{L_{i, i(f)}\right\}$ and $I_{G, V^{*}, 1}\left(M_{i, i(f)}\right)=\left\{P_{i, i(f)}, Y_{i, i(f)}\right\}$.

In both cases, $I_{G, V^{*}, 1}\left(Y_{i, i(f)}\right)=\left\{Y_{i, i(f)}\right\}$, and all vertices are 1-dominated and 1-separated by $V^{*}$ : we can conclude that $V^{*}$ is a rainbow 1-identifying set.

(b) Assume that there exists a rainbow 1-ID set $V^{*}$. Then we can still use the same argument as in the Cases (b) of the proofs of Propositions 10 and 16, in particular because every middle vertex $M_{i, i(f)}$ still needs to be 1-dominated by a codeword belonging to $W_{i, i(f)} \backslash\left\{M_{i, i(f)}\right\}$, in order to be 1-separated from $Y_{i, i(f)}$ by $V^{*}$. 


\subsection{Unique Rainbow 1-Dominating Codes}

We are going to prove that U-SAT and U-RDC $\mathrm{C}_{1}$ have equivalent complexities, up to polynomials; to this effect, we give a polynomial transformation from U-RDC ${ }_{1}$ to U-SAT (Proposition 20) and a polynomial transformation from U-3-SAT to U-RDC 1 (Proposition 21). One originality of this work is that we need to go both ways; in particular, we need the first transformation because U-SAT, or U-3-SAT, is not sufficiently well located inside DP.

Proposition 20 There exists a polynomial transformation from $U-R D C_{1}$ to U-SAT.

Proof. We start from an instance of $\mathrm{U}_{-} \mathrm{RDC}_{1}$, a coloured graph $G=(V, E)$ of order $n$, where $V=\left\{v_{1}, v_{2}, \ldots, v_{n}\right\}$; the set $\left\{c_{1}, c_{2}, \ldots, c_{\gamma}\right\}$ is the set of $\gamma$ colours used on $V$, and the number of occurrences of the colour $c_{i}$ is $\lambda_{i}$; we set $\Lambda_{i}=\Sigma_{j=1}^{i} \lambda_{j}$ for $1 \leq i \leq \gamma$. Without loss of generality, we can assume that $V_{1}=\left\{v_{1}, \ldots, v_{\Lambda_{1}}\right\}$ is the set of vertices with colour $c_{1}$, $V_{i}=\left\{v_{\Lambda_{i-1}+1}, \ldots, v_{\Lambda_{i}}\right\}$ the set of vertices with colour $c_{i}(2 \leq i \leq \gamma)$, so that the sets $V_{i}, 1 \leq i \leq \gamma$, partition $V$ and the vertices are ranked by increasing index of colour. For each vertex $v_{i}$, we denote by $v_{i}^{1}, \ldots, v_{i}^{s(i)}$ the $s(i)$ neighbours of $v_{i}$.

For each vertex $v_{i}$, we create the variable $x_{i}$. The set of clauses for U-SAT is constructed in the following way:

(a) for every $i \in\{1, \ldots, n\}$, we create the clause $\left\{x_{i}, x_{i}^{1}, \ldots, x_{i}^{s(i)}\right\}$;

(b1) for every $i \in\{1, \ldots, \gamma\}$, we create the clause $\left\{x_{j}: v_{j} \in V_{i}\right\}$;

(b2) for every $i \in\{1, \ldots, \gamma\}$ and for every pair of vertices $\left\{v_{p}, v_{q}\right\} \subseteq V_{i}$, $p<q$, we create the clause $\left\{\bar{x}_{p}, \bar{x}_{q}\right\}$.

Note that the number of variables and clauses is polynomial with respect to $n$, the order of $G$.

Now assume that we have a unique rainbow 1-dominating set $V^{*}$ in $G$. Define the assignment $\mathcal{A}_{1}$ on the variables $x_{i}$ by $\mathcal{A}_{1}\left(x_{i}\right)=\mathrm{T}$ if and only if $v_{i} \in V^{*}$. It is quite easy to see that the clauses described above are all satisfied: the clauses in (a) because $V^{*}$ is 1-dominating, the clauses in (b1) because every colour appears at least once in $V^{*}$, and the clauses in (b2) because every colour appears at most once in $V^{*}$.

Is $\mathcal{A}_{1}$ unique? Assume on the contrary that another assignment, $\mathcal{A}_{2}$, also satisfies the constructed instance of U-SAT, and define the vertex set $V^{+}$by the rule $v_{i} \in V^{+}$if and only if $\mathcal{A}_{2}\left(x_{i}\right)=\mathrm{T}$. Since $\mathcal{A}_{1} \neq \mathcal{A}_{2}$, we have $V^{+} \neq V^{*}$.

Because at least one literal is set TRUE by $\mathcal{A}_{2}$ in each clause from (a), every vertex $v_{i}$ is in $V^{+}$or has a neighbour in $V^{+}$, so the set $V^{+}$is 1dominating; the clauses in (b1), when satisfied by $\mathcal{A}_{2}$, show that every colour appears at least once in $V^{+}$and the clauses in (b2), that every colour appears at most once in $V^{+}$. Therefore, $V^{+}$is a rainbow 1-dominating set, but this contradicts the uniqueness of $V^{*}$. 

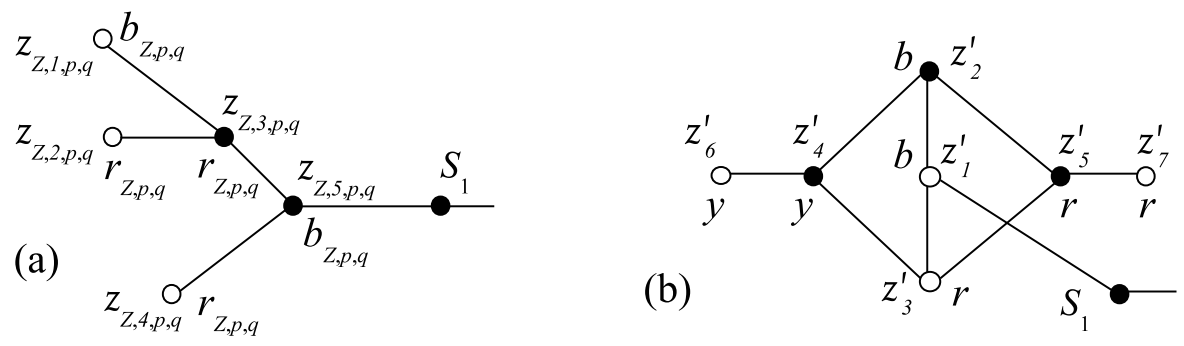

Figure 7: (a) The graph $H_{Z, p, q}$ for Proposition 21; (b) The graph $H_{Z, p, q}^{\prime}$ for Proposition 25, with lightened notation. Codewords are in black; all codewords are forced.

So a YES answer for U-RDC 1 leads to a YES answer for U-SAT. Assume now that the answer to $\mathrm{U}-\mathrm{RDC}_{1}$ is negative. If it is negative because there are at least two rainbow 1-D codes, then we have at least two assignments satisfying the instance of U-SAT: we have seen above how to construct a suitable assignment from a rainbow 1-D code, and different rainbow 1-D codes obviously lead to different assignments. If there is no rainbow 1$\mathrm{D}$ code, then there is no assignment satisfying U-SAT, because such an assignment would give a rainbow 1-D code, as we have seen above from $\mathcal{A}_{2}$. So in both cases, a NO answer to U-RDC 1 implies a NO answer to U-SAT.

Proposition 21 There exists a polynomial transformation from U-3-SAT to $U-R D C_{1}$. Moreover, in the connected graph constructed for this transformation, each colour appears at most thrice.

Proof. We consider again the construction for the proof of Proposition 10.

(i) We delete the vertices $z_{1}, z_{2}$ and the edges $z_{1} z_{2}$ and $z_{2} S_{1}$.

(ii) For each pair of antithetic literals $\ell_{p}, \ell_{q}$, we add one vertex $Z_{p, q}$ and the edges $v_{p} Z_{p, q}, Z_{p, q} v_{q}$, and set $\phi\left(Z_{p, q}\right)=b_{Z, p, q}$; since we assumed that each variable $x_{i}$ appears under its two forms, $x_{i}$ and $\bar{x}_{i}$, every clausal vertex is linked to at least one vertex of type $Z$. We then create the graph $H_{Z, p, q}$ with vertex set $V_{Z, p, q}=\left\{z_{Z, 1, p, q}, z_{Z, 2, p, q}, z_{Z, 3, p, q}, z_{Z, 4, p, q}, z_{Z, 5, p, q}\right\}$ and edge set $E_{Z, p, q}=\left\{z_{Z, 1, p, q} z_{Z, 3, p, q}, z_{Z, 2, p, q} z_{Z, 3, p, q}, z_{Z, 3, p, q} z_{Z, 5, p, q}, z_{Z, 4, p, q} z_{Z, 5, p, q}\right\}$, together with the edge $z_{Z, 5, p, q} S_{1}$. We set $\phi\left(z_{Z, 1, p, q}\right)=\phi\left(z_{Z, 5, p, q}\right)=b_{Z, p, q}$ and $\phi\left(z_{Z, 2, p, q}\right)=\phi\left(z_{Z, 3, p, q}\right)=\phi\left(z_{Z, 4, p, q}\right)=r_{Z, p, q}$. See Figure $7(\mathrm{a})$.

(iii) We give the colour $b_{M, i, i(f)}$ to every middle vertex $M_{i, i(f)} \in W_{i, i(f)}$ and we create the graph $H_{M, i, i(f)}$ which is identical to the graph $H_{Z, p, q}$, except that we replace $Z$ by $M, p$ by $i$ and $q$ by $i(f)$, cf. Figure 7(a).

The number of vertices linked to $S_{1}$, apart from $v_{1}$, is equal to the number of pairs of antithetic clausal vertices plus the number of gadgets, i.e., 1.5 
times the number of gadgets. One can already see that, in a rainbow 1D code, in $H_{Z, p, q}$ and $H_{M, i, i(f)}$, the vertices $z_{Z, 3, p, q}$ and $z_{Z, 5, p, q}$ on the one hand, the vertices $z_{M, 3, i, i(f)}$ and $z_{M, 5, i, i(f)}$ on the other hand, are necessarily codewords, and the other vertices are not. As a consequence, no middle vertex and no vertex $Z_{p, q}$ can belong to any rainbow 1-D code.

In the constructed graph, each colour appears at most thrice.

As in Case (b) of the proof of Proposition 10 about the consistency of the assignment defined by a rainbow $1-\mathrm{D}$ code, two antithetic causal vertices $v_{p}$ and $v_{q}$ cannot both be codewords, but, because $Z_{p, q}$ cannot belong to any rainbow 1-D code, one of them is a codeword; this extends to all the vertices antithetic to $v_{p}$, and so, all the clausal vertices corresponding to a variable $x$ belong to a rainbow 1-D code and none corresponding to $\bar{x}$, or the other way round.

(a) Assume that there is a unique assignment satisfying the instance of 3-SAT. We build the set $V^{*}$ in the following way: (i) for every true literal $\ell_{i}$, the clausal vertex $v_{i}$, with colour $i_{[0]}$, belongs to $V^{*}$; (ii) all the vertices $z_{Z, 3, p, q}, z_{Z, 5, p, q}, z_{M, 3, i, i(f)}$ and $z_{M, 5, i, i(f)}$, and all the unique vertices belong to $V^{*}$; (iii) for every true literal $\ell_{i}$ and $f \in\left\{1, \ldots, k_{i}\right\}$, the positive vertex $P_{i, i(f)} \in W_{i, i(f)}$ and the link vertex $L_{i, i(f)} \in W_{i, i(f)}$ belong to $V^{*}$; (iv) for every false literal $\ell_{i}$ and $f \in\left\{1, \ldots, k_{i}\right\}$, the negative vertex $N_{i, i(f)} \in W_{i, i(f)}$ belongs to $V^{*}$.

This is by now a routine task to check that $V^{*}$ is indeed a rainbow 1dominating set. What is new is that, after the step (i) has been performed, i.e., once the decision has been made for all the clausal vertices, there is no choice left for the completion of a rainbow 1-D code. To prove this claim, all we have to check are the gadgets, since we have already seen that the step (ii) is forced. Consider the literal $\ell_{1}$, its first antithetic literal $\ell_{1(1)}$, and the gadget $W_{1,1(1)}$, and assume first that $\ell_{1}=\mathrm{T}$. Then $v_{1} \in V^{*}$; this implies that $N_{1,1(1)} \notin V^{*}$, which implies in turn that $L_{1,1(1)} \in V^{*}, P_{1,1(1)} \in V^{*}$ and $N_{1,1(2)} \notin V^{*}$. So we can go on in the same way for $W_{1,1(2)}: L_{1,1(2)} \in V^{*}$, $P_{1,1(2)} \in V^{*}$ and $N_{1,1(3)} \notin V^{*}$, and so on until we reach $W_{1,1\left(k_{1}\right)}$, the last gadget for $\ell_{1}$. Here, we have $L_{1,1\left(k_{1}\right)} \in V^{*}, P_{1,1\left(k_{1}\right)} \in V^{*}$. We can repeat this argument for all the literals set TRUE by the assignment. Assume now that $\ell_{1}=\mathrm{F}$. Then all the antithetic vertices of $v_{1}$ are codewords, and for $1 \leq f \leq k_{1}$, all the link vertices $L_{1(f), 1}$ are codewords, none of the link vertices $L_{1,1(f)}$ are codewords, all the negative vertices $N_{1,1(f)}$ are codewords, none of the positive vertices $P_{1,1(f-1)}$ (when $f=1$, this is $v_{1}$ ) are codewords -and the vertex $P_{1,1\left(k_{1}\right)}$ is a codeword, because it is unique. This is true for all false literals, and so we had no choice for the positive, negative and link vertices (and we know from the beginning that the artificial vertices are codewords and the middle vertices are not).

Now assume that there exists another rainbow 1-dominating set, $V^{+}$. Then, denoting by $V_{\mathcal{C}}$ the set of clausal vertices, we have: $V_{\mathcal{C}} \cap V^{*} \neq V_{\mathcal{C}} \cap$ $V^{+}$. Setting the variable $x$ to TRUE if and only if one clausal vertex $v_{i}$ 
corresponding to one literal $\ell_{i}=x$ belongs to $V^{+}$(and we have seen that then all the literals equal to $x$ are in $V^{+}$, and those equal to $\bar{x}$ are not), we obtain a second valid assignment, which as before satisfies the instance of 3-SAT. This is a contradiction.

(b) Assume that the answer to U-3-SAT is NO, either because there is no assignment satisfying the instance, or because there are at least two such assignments. In the latter case, this would lead however to two different rainbow 1-dominating sets. On the other hand, if no assignment exists, then no rainbow 1-D code $V^{*}$ exists, because $V^{*}$ would lead, as before, to a valid assignment satisfying all the clauses - a contradiction.

Therefore, there is a YES answer to U-3-SAT if and only if there is a YES answer to U-RDC .

Remark 22 We can be more specific than in the last sentence of the proof above: actually, the proof shows that there is zero, one, or more than one solution to the instance of 3-SAT if and only if there is zero, one, or more than one solution, respectively, in the constructed coloured graph; this proves that this construction could also have been used to provide a polynomial transformation from 3-SAT to $R D C_{1}$, i.e., a proof of NP-completeness for $R D C_{1}$. This would not however give a result for paths, like Proposition 10, nor for trees where each colour appears at most twice, like Proposition 14.

Remark 23 If we allow unconnected graphs, then it is easy to build a graph where each colour appears at most twice, by using isolated vertices with a colour that we do not wish elsewhere: instead of building the graph $H_{Z, p, q}$ so that the vertex $Z_{p, q}$ with the colour $b_{Z, p, q}$ cannot be a codeword, we can simply create one isolated vertex with this colour. The same is true for $H_{M, i, i(f)}$.

\subsection{Unique Rainbow 1-Locating-Dominating Codes}

We have the same results for unique rainbow 1-LD codes as for unique rainbow 1-D codes.

Proposition 24 There exists a polynomial transformation from $U-R L D C_{1}$ to U-SAT.

Proof. The method is the same as in the proof of Proposition 20, and uses the characterization of LD codes in Remark 3, and in particular (2). Only the clauses in (a) will change, in order to fit the required locationdomination property: compared to the previous construction, the clauses in (b1) and (b2) are unchanged, and, keeping the same notation, the new clauses read:

(a1) for every $i \in\{1, \ldots, n\}$, we create the clause $\left\{x_{i}, x_{i}^{1}, \ldots, x_{i}^{s(i)}\right\}$ : here again, we translate the fact that we look for 1-dominating sets;

(a2) for each pair of vertices $v_{p}$ and $v_{q}$, we consider the set $B_{1}\left(v_{p}\right) \Delta$ $B_{1}\left(v_{q}\right)=\left\{v_{h_{1}}, v_{h_{2}}, \ldots, v_{h_{t}}\right\}$ (where $t$ depends on $v_{p}$ and $v_{q}$ ) and we construct 
the clause $\left\{x_{p}, x_{q}, x_{h_{1}}, x_{h_{2}}, \ldots, x_{h_{t}}\right\}$; we shall say that $\left\{x_{p}, x_{q}\right\}$ is the first part of the clause, and $\left\{x_{h_{1}}, x_{h_{2}}, \ldots, x_{h_{t}}\right\}$ its second part, which exists only when $t>0$ and may contain variables also appearing in the first part, a fact which is unimportant.

Assume that we have a unique rainbow 1-LD code, $V^{*}$; as previously, from $V^{*}$ we define an assignment $\mathcal{A}_{1}$. Then the clauses in (a1) are satisfied by $\mathcal{A}_{1}$, because $V^{*}$ is 1 -dominating. And the clauses in (a2) also are satisfied: if at least one of $v_{p}$ and $v_{q}$ is in $V^{*}$, then the first part of the clause contains a true literal; if neither $v_{p}$ nor $v_{q}$ is a codeword, then at least one vertex in $B_{1}\left(v_{p}\right) \Delta B_{1}\left(v_{q}\right)$ must be, and the second part of the clause contains a true literal.

The end of the proof is similar to the proof of Proposition 20.

Proposition 25 There exists a polynomial transformation from U-3-SAT to $U-R L D C_{1}$. Moreover, in the connected graph constructed for this transformation, each colour appears at most thrice.

Proof. Compared to the transformation from U-3-SAT to U-RDC 1 (Proposition 21), and after we have duplicated the vertices $S_{s}, 1 \leq s \leq m$, like we did for going from Proposition 10 to Proposition 16, the differences are:

(i) for each pair of antithetic literals $\ell_{p}, \ell_{q}$, we create three vertices $Z_{p, q}$, $Z_{p, q}^{\prime}$ and $Z_{p, q}^{\prime \prime}$, and the edges $v_{p} Z_{p, q}, Z_{p, q} Z_{p, q}^{\prime \prime}, Z_{p, q}^{\prime \prime} Z_{p, q}^{\prime}$, and $Z_{p, q}^{\prime} v_{q}$; the vertices $Z_{p, q}$ and $Z_{p, q}^{\prime}$ are given the colours $b_{Z, p, q}$ and $b_{Z^{\prime}, p, q}$, respectively, while $Z_{p, q}^{\prime \prime}$ is unique; then, to deal with $Z_{p, q}$, we create the graph $H_{Z, p, q}^{\prime}$ with vertex set $\left\{z_{Z, i, p, q}^{\prime}: 1 \leq i \leq 7\right\}$ represented in Figure $7(\mathrm{~b})$ where, for simplicity, we indicate only the second subscript of the vertices $z_{Z, i, p, q}^{\prime}$. The vertex $z_{Z, 1, p, q}^{\prime}$ is linked to $S_{1}$ and the colours are: $\phi\left(z_{Z, 1, p, q}^{\prime}\right)=\phi\left(z_{Z, 2, p, q}^{\prime}\right)=b_{Z, p, q}$, $\phi\left(z_{Z, 3, p, q}^{\prime}\right)=\phi\left(z_{Z, 5, p, q}^{\prime}\right)=\phi\left(z_{Z, 7, p, q}^{\prime}\right)=r_{Z, p, q}$ and $\phi\left(z_{Z, 4, p, q}^{\prime}\right)=\phi\left(z_{Z, 6, p, q}^{\prime}\right)=$ $y_{Z, p, q}$ (for Yellow). Again for simplicity, we drop the subscripts of the colours in Figure 7(b). Similarly, we create the graph $H_{Z^{\prime}, p, q}^{\prime}$ for $Z_{p, q}^{\prime}$.

(ii) We give the colour $b_{M, i, i(f)}$ to every middle vertex $M_{i, i(f)} \in W_{i, i(f)}$ and we create the graph $H_{M, i, i(f)}^{\prime}$ which is identical to the graph $H_{Z, p, q}^{\prime}$, except that we replace $Z$ by $M, p$ by $i$ and $q$ by $i(f)$, cf. Figure $7(\mathrm{~b})$.

Remark 26 Assume that $V^{*}$ is a rainbow 1-LD code. Because $z_{Z, 2, p, q}^{\prime}$ and $z_{Z, 3, p, q}^{\prime}$ have the same neighbours, at least one of them must belong to $V^{*}$; if however $z_{Z, 3, p, q}^{\prime} \in V^{*}$, then $z_{Z, 7, p, q}^{\prime}$ cannot be 1-dominated by $V^{*}$. So $z_{Z, 2, p, q}^{\prime} \in V^{*}, z_{Z, 3, p, q}^{\prime} \notin V^{*}$, and $z_{Z, 1, p, q}^{\prime} \notin V^{*}$. It is then straightforward to check that necessarily the Yellow and Red codewords are $z_{Z, 4, p, q}^{\prime}$ and $z_{Z, 5, p, q}^{\prime}$, respectively (and $z_{Z, 6, p, q}^{\prime} \notin V^{*}, z_{Z, 7, p, q}^{\prime} \notin V^{*}$ ).

As a consequence, no middle vertex and no vertex $Z_{p, q}, Z_{p, q}^{\prime}$ can belong to any rainbow $1-L D$ code.

In the constructed graph, each colour appears at most thrice.

As in Case (b) of the proof of Proposition 21 about the consistency of the assignment defined by a rainbow $1-\mathrm{D}$ code, two antithetic causal vertices 
$v_{p}$ and $v_{q}$ cannot both be codewords; on the other hand, because neither $Z_{p, q}$ nor $Z_{p, q}^{\prime}$ can belong to any rainbow 1-LD code, one of $v_{p}$ and $v_{q}$ is a codeword, so that $Z_{p, q}$ and $Z_{p, q}^{\prime}$ are 1 -separated by a codeword. This extends to all the vertices antithetic to $v_{p}$; so, all the clausal vertices corresponding to a variable $x$ belong to a rainbow 1-LD code and none corresponding to $\bar{x}$, or the other way round.

The proof then follows the lines of the proof of Proposition 21.

\subsection{Unique Rainbow 1-Identifying Codes}

We obtain a better result for unique rainbow 1-ID codes, in the sense that, starting from U-3-SAT, we are able to construct a coloured graph where each colour appears at most twice. But first we go from U-RIDC H $_{1}$ to U-SAT:

Proposition 27 There exists a polynomial transformation from $U-R I D C_{1}$ to U-SAT.

Proof. Same technique as for Propositions 20 and 24. We start from a twin-free graph and define the clauses of type (a) as follows (it relies on the characterization of Remark 3):

(a1) for every $i \in\{1, \ldots, n\}$, we create the clause $\left\{x_{i}, x_{i}^{1}, \ldots, x_{i}^{s(i)}\right\}$;

(a2) for each pair of vertices $v_{p}$ and $v_{q}$, we consider the set $B_{1}\left(v_{p}\right) \Delta$ $B_{1}\left(v_{q}\right)=\left\{v_{h_{1}}, v_{h_{2}}, \ldots, v_{h_{t}}\right\}$; because $G$ is twin-free, we have $t>0$. Then we construct the clause $\left\{x_{h_{1}}, x_{h_{2}}, \ldots, x_{h_{t}}\right\}$, which is simply the second part of the clause defined in the Case (a2) of the proof of Proposition 24.

The end of the proof is similar to the previous two proofs of this type.

Proposition 28 There exists a polynomial transformation from U-3-SAT to $U-R I D C_{1}$. Moreover, in the connected graph constructed for this transformation, each colour appears at most twice.

Proof. With respect to the original construction, we triplicate the vertices $S_{s}, 1 \leq s \leq m+1$, all the artificial vertices, and the final vertex $J$. For each pair of antithetic literals $\ell_{p}, \ell_{q}$, we add two vertices $Z_{p, q}, Z_{p, q}^{\prime}$ and the edges $v_{p} Z_{p, q}, Z_{p, q} v_{q}$ and $Z_{p, q} Z_{p, q}^{\prime}$, and set $\phi\left(Z_{p, q}\right)=b_{Z, p, q}$; the vertex $Z_{p, q}^{\prime}$ is unique. We give the colour $b_{M, i, i(f)}$ to every middle vertex $M_{i, i(f)} \in W_{i, i(f)}$. For each middle vertex $M_{i, i(f)}$, we create the unique vertex $Y_{i, i(f)}$, which is linked to $M_{i, i(f)}$.

For every pair of antithetic literals $\ell_{p}, \ell_{q}$, we create the graph $H_{Z, p, q}^{\prime}$ given by Figure $5(\mathrm{~b})$, with the edge $z_{Z, 1, p, q}^{\prime} S_{1}$, and for every middle vertex $M_{i, i(f)}$, the similar graph $H_{M, i, i(f)}^{\prime}$ and the edge $z_{M, 1, i, i(f)}^{\prime} S_{1}$.

We have already observed that $z_{Z, 2, p, q}^{\prime}$ and $z_{Z, 3, p, q}^{\prime}$, not $z_{Z, 1, p, q}^{\prime}$, necessarily belong to any rainbow 1-ID code, and the same is true for $z_{M, 2, i, i(f)}^{\prime}$ and $z_{M, 3, i, i(f)}^{\prime}$, and $z_{M, 1, i, i(f)}^{\prime}$. Then one can see that exactly one of the two antithetic vertices, $v_{p}$ or $v_{q}$, belongs to any rainbow 1-ID code. 
The end of the proof is the same as that of Propositions 21 and 25.

\subsection{Generalization to $r>1$ : Dominating Sets}

We start with an easy lemma, which is actually common to the three problems.

Lemma 29 Let $r \geq 2$ be any integer. There is a polynomial transformation from $U-R D C_{r}$ to $U-S A T$, from $U-R L D C_{r}$ to $U$-SAT, and from $U-R I D C_{r}$ to $U-S A T$.

Proof. Let $G=(V, E)$ be a coloured graph, assumed to be $r$-twin-free in the case of U-RIDC ${ }_{r}$, and consider $G^{r}$, the $r$-th power of $G$. By Remark 2, there is a unique rainbow 1-D code (respectively, 1-LD code, 1-ID code) in $G^{r}$ if and only if there is a unique rainbow $r$-D code (respectively, $r$-LD code, $r$-ID code) in $G$. Therefore, we have a first transformation, from $\mathrm{U}_{-} \mathrm{RDC}_{r}$

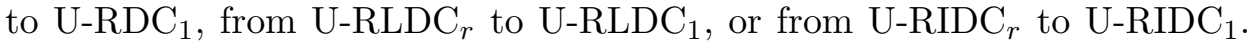
Then we apply Propositions 20, 24 and 27, with their transformations from $\mathrm{U}_{-\mathrm{RDC}}{ }_{1}$ to U-SAT, from U-RLDC 1 to U-SAT, from U-RIDC ${ }_{1}$ to U-SAT, and the transitivity of polynomial transformations.

Then we turn to rainbow $r$-dominating sets.

Proposition 30 Let $r \geq 2$ be any integer. There is a polynomial transformation from $U-R D C_{1}$ to $U-R D C_{r}$.

Proof. Let $G=(V, E)$ be any instance of $\mathrm{U}_{-\mathrm{RDC}_{1}}$, that is, any coloured graph, and let $e=v_{1} v_{2}$ be any edge in $G$. Let

$$
\begin{gathered}
\quad V_{e}^{\S}=\left\{\alpha_{e, i}: 1 \leq i \leq r-1\right\} \cup\left\{\beta_{e, i, j}: 1 \leq i \leq r-1,1 \leq j \leq 3 r\right\}, \\
E_{e}^{\S}=\left\{v_{1} \alpha_{e, 1}, \alpha_{e, 1} \alpha_{e, 2}, \ldots, \alpha_{e, r-2} \alpha_{e, r-1}, \alpha_{e, r-1} v_{2}\right\} \cup \\
\left\{\alpha_{e, i} \beta_{e, i, 1}, \beta_{e, i, 1} \beta_{e, i, 2}, \ldots, \beta_{e, i, r} \beta_{e, i, r+1}, \ldots, \beta_{e, i, 2 r-1} \beta_{e, i, 2 r}: 1 \leq i \leq r-1\right\} \\
\cup\left\{\beta_{e, i, r} \beta_{e, i, 2 r+1}, \beta_{e, i, 2 r+1} \beta_{e, i, 2 r+2}, \ldots, \beta_{e, i, 3 r-1} \beta_{e, i, 3 r}: 1 \leq i \leq r-1\right\},
\end{gathered}
$$

see Figure 8. For $i \in\{1, \ldots, r-1\}$, the vertex $\alpha_{e, i}$ and all the vertices $\beta_{e, i, j}$, $1 \leq j \leq 3 r$, (i.e., the branch linked to $\alpha_{e, i}$ ) receive the colour $b_{i, e}$, which will appear nowhere else. Then we set

$$
V^{\S}=V \cup\left(\cup_{e \in E} V_{e}^{\S}\right), E^{\S}=\cup_{e \in E} E_{e}^{\S},
$$

and $G^{\S}=\left(V^{\S}, E^{\S}\right)$ is the instance of $\mathrm{U}_{-} \mathrm{RDC}_{r}$ (the colours in $V$ remain the same). Note in particular that any two vertices at distance 1 in $G$ are at distance $r$ in $G^{\S}$; this is why we shall say that the edge $e$ is dilated.

We claim that an instance of $\mathrm{U}_{-} \mathrm{RDC}_{1}$ is positive if and only if the corresponding constructed instance of $\mathrm{U}-\mathrm{RDC}_{r}$ is.

(a) First, we assume that there is a YES answer for $\mathrm{U}_{-} \mathrm{RDC}_{1}$ : there is a unique rainbow 1-dominating code $V_{1}^{*}$ in $G$. Let $W$ be the set consisting of 


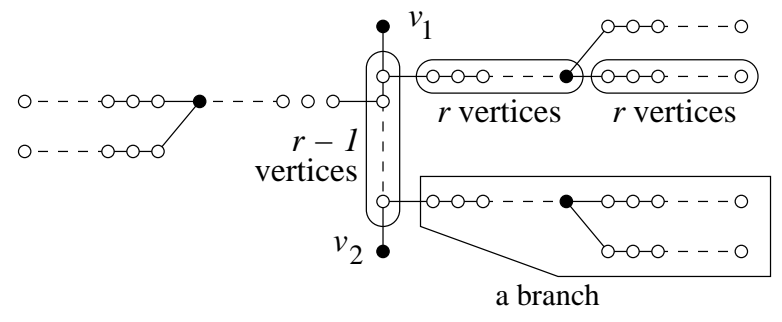

Figure 8: How the edge $e=v_{1} v_{2} \in E$ gives $V_{e}^{\S}$ and $E_{e}^{\S}$. The black vertices on the branches are the vertices $\beta_{e, i, r}$.

the $(r-1)|E|$ vertices $\beta_{e, i, r}, e \in E, 1 \leq i \leq r-1$ (they are represented as black vertices in Figure 8). Note that $W r$-dominates exactly $V^{\S} \backslash V$. Then $V_{1}^{+}=V_{1}^{*} \cup W$ is a rainbow $r$-dominating set in $G^{\S}$. Is $V_{1}^{+}$unique?

Assume on the contrary that $V_{2}^{+}$is another rainbow $r$-D code in $G^{\S}$. Because $\beta_{e, i, r}$ is the only vertex $r$-dominating the two extremities of its branch, $\beta_{e, i, 2 r}$ and $\beta_{e, i, 3 r}$, and for rainbow reasons, we have

$$
\left(V^{\S} \backslash V\right) \cap V_{2}^{+}=W .
$$

Let $V_{2}^{*}=V_{2}^{+} \backslash W=V_{2}^{+} \cap V$. Clearly, $V_{2}^{*}$ is a rainbow 1-D code in $G$, different from $V_{1}^{*}$, a contradiction.

(b) Next, we assume that the answer to $\mathrm{U}_{-} \mathrm{RDC}_{1}$ is NO: either there is no rainbow 1-dominating code in $G$, or there is more than one. In the latter case, we have more than one rainbow $r$-D code in $G^{\S}$ : simply add the set $W$ to the codes in $G$. So we assume that we are in the first case. If there is a rainbow $r$-D code $V^{+}$in $G^{\S}$, then again $V^{+} \backslash W$ would be a rainbow 1-D code in $G$, a contradiction. In all cases, the answer to $\mathrm{U}_{-} \mathrm{RDC}_{r}$ is also NO.

Corollary 31 Let $r \geq 1$ be any integer. The decision problem $R D C_{r}$ is NP-complete.

Proof. We can use Remark 22 here: the above proof of Proposition 30 shows that there is zero, one, or more than one solution to the instance of $\mathrm{U}_{-} \mathrm{RDC}_{1}$ if and only if there is zero, one, or more than one solution, respectively, in the constructed coloured graph $G^{\S}$; this proves that we have a polynomial transformation from $\mathrm{RDC}_{1}$ to $\mathrm{RDC}_{r}$, i.e., a proof of $N P$-completeness for $\mathrm{RDC}_{r}$, since $\mathrm{RDC}_{1}$ is $N P$-complete (Propositions 10 or 14 ) and $\mathrm{RDC}_{r}$ obviously belongs to $N P$.

However, a better result can be obtained.

Proposition 32 Let $r \geq 1$ be any integer. The decision problem $R D C_{r}$ is NP-complete, even when restricted to coloured trees. 


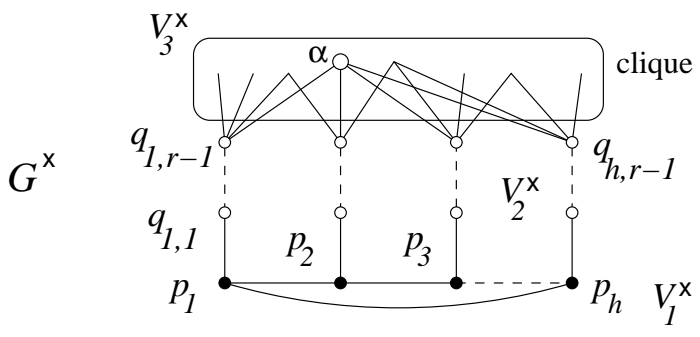

Figure 9: The graph $G^{\times}$. Black vertices are forced codewords. White vertices are forced non-codewords.

Proof. Apply the construction from the proof of Proposition 30, which yields a tree, to the path constructed in the proof of Proposition 10.

For $r>1$, we do not have results with a fixed number of occurrences of the colours. We can now conclude for the problem $\mathrm{U}-\mathrm{RDC}_{r}$.

Proposition 33 Let $r \geq 1$ be any integer. The decision problems U-SAT and $U-R D C_{r}$ have equivalent complexities, up to polynomials.

Proof. There is a polynomial transformation from U-3-SAT to U-RDC 1 (Proposition 21), from U-RDC 1 to $\mathrm{U}_{-} \mathrm{RDC}_{r}$ (Proposition 30), and from U$\mathrm{RDC}_{r}$ to U-SAT (Lemma 29).

\subsection{Generalization to $r>1$ : Locating-Dominating Sets}

In order to dilate the edges, we need, as with the branches and the set $W$ in the case of dominating codes, a graph which has all its codewords (and non-codewords) forced.

Let $r \geq 2$ and $h=2 r+1$. Let $G_{1}^{\times}=\left(V_{1}^{\times}, E_{1}^{\times}\right)$be the cycle of length $h$, with $V_{1}^{\times}=\left\{p_{i}: 1 \leq i \leq h\right\}$. Then we construct $G_{2}^{\times}=\left(V_{2}^{\times}, E_{2}^{\times}\right)$, with $V_{2}^{\times}=$ $\left\{q_{i, j}: 1 \leq i \leq h, 1 \leq j \leq r-1\right\}$ and $E_{2}^{\times}=\cup_{1 \leq i \leq h}\left\{q_{i, j} q_{i, j+1}: 1 \leq j \leq r-2\right\}$. The set of edges between $G_{1}^{\times}$and $G_{2}^{\times}$is $E_{1,2}^{\times}=\left\{p_{i} q_{i, 1}: 1 \leq i \leq h\right\}$. Next, we construct $G_{3}^{\times}=\left(V_{3}^{\times}, E_{3}^{\times}\right)$with $V_{3}^{\times}=\left\{s_{i}: 1 \leq i \leq 2^{h}-1-(r-1) h\right\}$ and $E_{3}^{\times}=\left\{s_{i_{1}} s_{i_{2}}: 1 \leq i_{1}<i_{2} \leq\left|V_{3}^{\times}\right|\right\}$, i.e., $G_{3}^{\times}$is a clique.

We set $V^{\times}=V_{1}^{\times} \cup V_{2}^{\times} \cup V_{3}^{\times}$, see Figure 9 .

In order to define the set $E_{2,3}^{\times}$of edges between $\left\{q_{i, r-1}: 1 \leq i \leq h\right\}$ and $V_{3}^{\times}$, we introduce, for every vertex $v \in V_{2}^{\times} \cup V_{3}^{\times}$, the signature of $v$ as the set $B_{r}(v) \cap V_{1}^{\times}$of the elements of the cycle that $r$-dominate $v$, and we wish to have nonempty and distinct signatures. Since

(a) the $h$ vertices in $V_{1}^{\times}$can provide $2^{h}-1$ such signatures;

(b) $\left|V_{2}^{\times} \cup V_{3}^{\times}\right|=\left|V_{2}^{\times}\right|+\left|V_{3}^{\times}\right|=2^{h}-1$;

(c) because $h$ is sufficiently large, the vertices $q_{i, j}$ in $V_{2}^{\times}$have nonempty and different signatures (of odd size $2 r-2 j+1 \geq 3$ ); 
(d) a vertex in $V_{3}^{\times}$which is linked (respectively, not linked) to $q_{i, r-1}$ is at distance equal to (respectively, greater than) $r$ from $p_{i}$;

we can see that it is possible to construct $E_{2,3}^{\times}$in such a way that the vertices in $V_{3}^{\times}$have nonempty signatures which are different inside $V_{3}^{\times}$, and different from those for $V_{2}^{\times}$. In particular, in $V_{3}^{\times}$there is a vertex which has signature equal to $V_{1}^{\times}$; we denote this vertex by $\alpha$. Note also that we could not have more vertices with this signature property.

We set $E^{\times}=E_{1}^{\times} \cup E_{1,2}^{\times} \cup E_{2}^{\times} \cup E_{2,3}^{\times} \cup E_{3}^{\times}$and $G^{\times}=\left(V^{\times}, E^{\times}\right)$. The order of $G^{\times}$is $n^{\times}=2^{h}-1+h$. Then we give colours to $G^{\times}$in the following way: the vertices in $V_{1}^{\times} \backslash\left\{p_{r+1}\right\}$ are unique, the other vertices share the Blue colour.

Lemma 34 The only rainbow $r-L D$ code in $G^{\times}$is the cycle $V_{1}^{\times}$.

Proof. Every rainbow $r$-LD code must contain the $h-1$ unique vertices, plus one Blue vertex. Consider the four vertices $w_{1}, w_{2}, w_{3}, w_{4}$ in $V_{3}^{\times}$with signatures $\left\{p_{1}\right\},\left\{p_{2}\right\},\left\{p_{1}, p_{r+1}\right\}$ and $\left\{p_{2}, p_{r+1}\right\}$, respectively. No vertex in $V_{3}^{\times}$, no vertex in $V_{2}^{\times}$, can $r$-separate $w_{1}$ and $w_{3}, w_{2}$ and $w_{4}$, only $p_{r+1}$ can. On the other hand, $V_{1}^{\times}$is a rainbow $r$-LD code, because now the signatures are simply the sets $I_{G^{\times}, V_{1}^{\times}, r}(v)$, for $v \in V_{2}^{\times} \cup V_{3}^{\times}=V^{\times} \backslash V_{1}^{\times}$: by construction, they are all nonempty and distinct.

Proposition 35 Let $r \geq 2$ be any integer. There is a polynomial transformation from $U-R L D C_{1}$ to $U-R L D C_{r}$.

Proof. We start from an instance of $\mathrm{U}^{-R L D C} \mathrm{C}_{1}$, i.e., a coloured graph $G=(V, E)$ of order $n$.

For each edge $e=v_{1} v_{2} \in E$ that we want to dilate, we "paste" $r-1$ copies of the graph $G^{\times}$, by deleting the edge $e=v_{1} v_{2}$ and creating the edges $v_{1} \alpha_{1}, \alpha_{1} \alpha_{2}, \ldots, \alpha_{r-1} v_{2}$, where the $\alpha_{i}$ 's are copies of the vertex $\alpha$ in $G^{\times}$; see Figure 10. We denote by $G^{\S}=\left(V^{\S}, E^{\S}\right)$ the graph thus constructed. The colours in $G^{\S}$ are given as follows: the colours in $V$ are unchanged, the vertices in each copy of $V_{1}^{\times} \backslash\left\{p_{r+1}\right\}$ are unique, and the other vertices in each copy of $G^{\times}$share the colour $b_{i}$, i.e., one different colour for each copy.

The order of $G^{\S}$ is $|V|+|E|(r-1)\left(2^{h}+h-1\right)$. Since $r$, hence $h=2 r+1$, is fixed, this does not affect the polynomiality of our construction with respect to $n$, the order of $G$.

We claim that there is a unique rainbow 1-LD code in $G$ if and only if there is a unique rainbow $r$-LD code in $G^{\S}$.

(a) Assume first that there is a unique rainbow $1-\mathrm{LD}$ code $V_{1}^{*}$ in $G$. We construct the following code $V_{1}^{+}$in $G^{\S}$ : we add to $V_{1}^{*}$ the set $W$ of all the vertices in all the cycles $G_{1}^{\times}$in all the copies of $G^{\times}$. Note that these vertices in $W$ do not $r$-dominate any vertex in $V$. Obviously, $V_{1}^{+}$is a rainbow $r$-LD code in $G^{\S}$. Is it unique? 


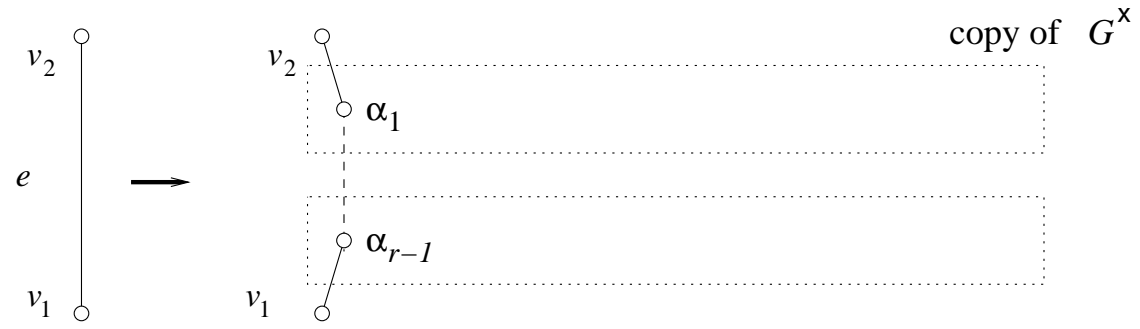

Figure 10: How the edge $e=v_{1} v_{2} \in E$ is dilated for Proposition 35 .

The argument is similar to the one for dominating codes: assume on the contrary that $V_{2}^{+}$is another rainbow $r$-LD code in $G^{\S}$. The intersection of $V^{\S} \backslash V$ with $V_{2}^{+}$is equal to $W$, because, since the vertices in $V$ do not $r$ separate between any vertices in any copy of the clique $G_{3}^{\times}$, except possibly the vertex $\alpha$, we can still apply the argument of Lemma 34, and it is still true that, in addition to the unique vertices, we must take as codewords all the copies $p_{r+1}^{i}, 1 \leq i \leq|E|(r-1)$, of the vertex $p_{r+1}$. Let $V_{2}^{*}=V_{2}^{+} \backslash W=$ $V_{2}^{+} \cap V$. Clearly, $V_{2}^{*}$ is a rainbow 1-LD code in $G$, different from $V_{1}^{*}$, a contradiction.

(b) Next, we assume that the answer to U-RLDC 1 is NO: either there is no rainbow 1-LD code in $G$, or there is more than one. In the latter case, we have more than one rainbow $r$-LD code in $G^{\S}$ : simply add the set $W$ to the codes in $G$. So we assume that we are in the first case. But if there is a rainbow $r$-LD code $V^{+}$in $G^{\S}$, then again $V^{+} \backslash W$ would be a rainbow 1 -LD code in $G$, a contradiction. In both cases, the answer to U-RLDC $r$ is also NO.

The following consequences are immediate.

Corollary 36 Let $r \geq 1$ be any integer. The decision problem $R L D C_{r}$ is NP-complete.

Proposition 37 Let $r \geq 1$ be any integer. The decision problems U-SAT and $U-R L D C_{r}$ have equivalent complexities, up to polynomials.

\subsection{Generalization to $r>1$ : Identifying Sets}

Lemma 38 Let $r \geq 1$ be any integer. Let $G=(V, E)$ be the (non coloured) path $\beta_{1} \beta_{2} \ldots \beta_{3 r+1}$. Then $V^{*}=\left\{\beta_{r+1}, \ldots, \beta_{2 r}\right\}$ is included in any $r$-identifying code.

Proof. Apply Lemma 4 in [11] with $n=3 r+1$. Note that $\beta_{2 r+1}$ also belongs to any $r$-identifying code, but we do not need it for our purpose. $\triangle$

We colour the previous path in the following way: for $i \in\{1, \ldots, r\}, \phi\left(\beta_{i}\right)=$ $\phi\left(\beta_{i+r}\right)=c_{i}$; the vertices $\beta_{i}, 2 r+1 \leq i \leq 3 r+1$, are unique. 
Lemma 39 The coloured path defined above admits $V^{+}=\left\{\beta_{i}: r+1 \leq i \leq\right.$ $3 r+1\}$ as its unique rainbow $r$-identifying code.

Proof. A rainbow $r$-ID code must contain $V^{*}$ and the unique vertices. The vertices $\beta_{i}, 1 \leq i \leq r$, cannot be codewords for rainbow reasons. It is easy to check that $V^{+}$is indeed a rainbow $r$-ID code, and is the only one.

Proposition 40 Let $r \geq 2$ be any integer. There is a polynomial transformation from $U-R I D C_{1}$ to $U-R I D C_{r}$.

Proof. Let $G=(V, E)$ be any instance of $\mathrm{U}_{-R I D C}$, that is, any coloured graph, and let $e=v_{1} v_{2}$ be any edge in $G$. Let

$$
\begin{gathered}
V_{e}^{\S}=\left\{\beta_{e, i, j}: 1 \leq i \leq r-1,1 \leq j \leq 3 r+1\right\}, \\
E_{e}^{\S}=\left\{v_{1} \beta_{e, 1,1}, \beta_{e, 1,1} \beta_{e, 2,1}, \ldots, \beta_{e, r-2,1} \beta_{e, r-1,1}, \beta_{e, r-1,1} v_{2}\right\} \cup \\
\left\{\beta_{e, i, 1} \beta_{e, i, 2}, \beta_{e, i, 2} \beta_{e, i, 3}, \ldots, \beta_{e, i, 3 r} \beta_{e, i, 3 r+1}: 1 \leq i \leq r-1\right\},
\end{gathered}
$$

i.e., in order to dilate all the edges, we have split every edge $e$ with $r-1$ vertices $\beta_{e, i, 1}$ which are the starting points of copies of the path defined for Lemma 38. Then we set

$$
V^{\S}=V \cup\left(\cup_{e \in E} V_{e}^{\S}\right), E^{\S}=\cup_{e \in E} E_{e}^{\S},
$$

and $G^{\S}=\left(V^{\S}, E^{\S}\right)$. The set $V$ keeps its colours unchanged. Each path is coloured as was done for Lemma 39, with its specific colours that will be nowhere else. Note that the set $\left\{\beta_{e, i, j}: r+1 \leq j \leq 3 r+1\right\} r$-dominates exactly the set $\left\{\beta_{e, i, j}: 1 \leq j \leq 3 r+1\right\}$, for all $e$ and $i$.

We claim that the instance of U-RIDC 1 we started from, is positive if and only if the coloured graph $G^{\S}$ admits a unique rainbow $r$-ID code.

(a) First, we assume that there is a unique rainbow 1-ID code $V_{1}^{*}$ in $G$. Let $W$ be the set consisting of the $|E|(2 r+1)(r-1)$ vertices $\beta_{e, i, j}, e \in E$, $1 \leq i \leq r-1, r+1 \leq j \leq 3 r+1$. Note that $W r$-dominates exactly $V^{\S} \backslash V$. Then $V_{1}^{+}=V_{1}^{*} \cup W$ is a rainbow $r$-identifying set in $G^{\S}$. Is $V_{1}^{+}$unique?

Assume on the contrary that $V_{2}^{+}$is another such code in $G^{\S}$. We have $\left(V^{\S} \backslash V\right) \cap V_{2}^{+}=W$. Let $V_{2}^{*}=V_{2}^{+} \backslash W=V_{2}^{+} \cap V$. Clearly, $V_{2}^{*}$ is a rainbow 1-ID code in $G$, different from $V_{1}^{*}$, a contradiction.

(b) Next, we assume that the answer to U-RIDC $\mathrm{U}_{1}$ is NO: either there is no rainbow 1-ID code in $G$, or there is more than one. In the latter case, we have more than one rainbow $r$-ID code in $G^{\S}$ : simply add the set $W$ to the codes in $G$. So we assume that we are in the first case. If there is a rainbow $r$-ID code $V^{+}$in $G^{\S}$, then again $V^{+} \backslash W$ would be a rainbow 1-ID code in $G$, a contradiction. In both cases, the answer to $\mathrm{U}_{-\mathrm{RIDC}}$ is $\mathrm{NO}$.

As previously, we have the following easy consequences.

Corollary 41 Let $r \geq 1$ be any integer. The decision problem $R I D C_{r}$ is NP-complete. 
Proposition 42 Let $r \geq 1$ be any integer. The decision problem $R I D C_{r}$ is NP-complete, even when restricted to coloured trees.

Proposition 43 Let $r \geq 1$ be any integer. The decision problems U-SAT and $U-R I D C_{r}$ have equivalent complexities, up to polynomials.

\section{Conclusion}

We recapitulate all the above results and summarize them in Table 1 below.

(i) The decision problem $\mathrm{RDC}_{1}$ was known to be $N P$-complete, even for paths [1], [2]. We proved that it is $N P$-complete, even when restricted to trees where each colour appears at most twice (Proposition 14). For all $r \geq 2$, we proved that the decision problem $\mathrm{RDC}_{r}$ is $N P$-complete, even for trees (Proposition 32).

We proved that the decision problems $\mathrm{U}_{-} \mathrm{RDC}_{1}$ and U-SAT have equivalent complexities, up to polynomials, and settled the case when each colour appears at most thrice (Propositions 20 and 21). For all $r \geq 2$, we proved that the decision problems U-RDC $r$ and U-SAT have equivalent complexities, up to polynomials (Proposition 33). Using Corollary 6, we have the following result.

Proposition 44 For all $r \geq 1$, the decision problem $U-R D C_{r}$ is $N P$-hard and belongs to the class DP.

(ii) We proved that the decision problem $\mathrm{RLDC}_{1}$ is $N P$-complete, even for paths (Proposition 16), and when restricted to trees where each colour appears at most twice (Proposition 17). For all $r \geq 2$, we proved that the decision problem $\mathrm{RLDC}_{r}$ is $N P$-complete (Corollary 36 ).

We proved that the decision problems $\mathrm{U}_{-\mathrm{RLDC}}$ and U-SAT have equivalent complexities, up to polynomials, and settled the case when each colour appears at most thrice (Propositions 24 and 25). For all $r \geq 2$, we proved that the decision problems U-RLDC $r$ and U-SAT have equivalent complexities, up to polynomials (Proposition 37). Using Corollary 6, we have the following result.

Proposition 45 For all $r \geq 1$, the decision problem $U-R L D C_{r}$ is NP-hard and belongs to the class DP.

(iii) We proved that the decision problem $\mathrm{RIDC}_{1}$ is $N P$-complete, even when restricted to trees where each colour appears at most twice (Proposition 19). For all $r \geq 2$, we proved that the decision problem $\mathrm{RIDC}_{r}$ is $N P$-complete, even for trees (Proposition 42).

We proved that the decision problems $\mathrm{U}_{-\mathrm{RIDC}_{1}}$ and U-SAT have equivalent complexities, up to polynomials, and settled the case when each colour appears at most twice (Propositions 27 and 28). For all $r \geq 2$, we proved 
that the decision problems U-RIDC $r$ and U-SAT have equivalent complexities, up to polynomials (Proposition 43). Using Corollary 6, we have the following result.

Proposition 46 For all $r \geq 1$, the decision problem $U$-RIDC $C_{r}$ is NP-hard and belongs to the class DP.

In Remark 7, it is said that "U-SAT is not believed to be DP-complete", so the same can be said for U-RDC $r$, U-RLDC $r$, and U-RIDC $r$.

\begin{tabular}{|l||l|l||l|l||l|l|}
\hline \multicolumn{1}{|c||}{} & \multicolumn{2}{c||}{$r$-D codes } & \multicolumn{2}{c||}{$r$-LD codes } & $r$-ID codes \\
\hline \hline$N P$-complete, $r=1$ & $\begin{array}{l}\text { path }[1],[2] \\
\text { tree }\end{array}$ & $\begin{array}{l}? \\
\leq 2\end{array}$ & $\begin{array}{l}\text { path } \\
\text { tree }\end{array}$ & $\begin{array}{l}? \\
\leq 2\end{array}$ & tree & $\leq 2$ \\
\hline$N P$-complete, $r>1$ & tree & $?$ & graph & $?$ & tree & $?$ \\
\hline \hline uniqueness: $\approx$ U-SAT, $r=1$ & graph & $\leq 3$ & graph & $\leq 3$ & graph & $\leq 2$ \\
\hline uniqueness: $\approx$ U-SAT, $r>1$ & graph & $?$ & graph & $?$ & graph & $?$ \\
\hline
\end{tabular}

Table 1: The $2^{\text {nd }}, 4^{\text {th }}$, and $6^{\text {th }}$ columns give the structure of the graph, the $3^{\text {rd }}, 5^{\text {th }}$, and $7^{\text {th }}$ columns give the maximum number of colour occurrences.

\section{References}

[1] J.-A. ANGLÈS d'AURIAC, Cs. BUJTÁS, A. El MAFTOUHI, M. KARPINSKI, Y. MANOUSSAKIS, L. MONTERO, N. NARAYANAN, L. ROSAZ, J. THAPPER and ZS. TUZA: Tropical dominating sets in vertex-coloured graphs, Proceedings of 10th International Workshop on Algorithms and Computation, Kathmandu, Nepal, pp. 17-27, 2016.

[2] J.-A. ANGLÈS d'AURIAC, Cs. BUJTÁS, A. El MAFTOUHI, M. KARPINSKI, Y. MANOUSSAKIS, L. MONTERO, N. NARAYANAN, L. ROSAZ, J. THAPPER and ZS. TUZA: Tropical dominating sets in vertex-coloured graphs, Journal of Discrete Algorithms, Vol. 48, pp. 27-41, 2018.

[3] J.-A. ANGLÈS d'AURIAC, N. COHEN, A. El MAFTOUHI, A. HARUTYUNYAN, S. LEGAY, and Y. MANOUSSAKIS: Connected tropical subgraphs in vertex-colored graphs, Discrete Mathematics 85 Theoretical Computer Science, Vol. 17(3), P21, 2016.

[4] J.-P. BARThÉLEMY, G. D. COHEN and A. C. LOBSTEIN: Algorithmic Complexity and Communication Problems, London: University College of London, 1996.

[5] A. BLASS and Y. GUREVICH: On the unique satisfiability problem, Information and Control, Vol. 55, pp. 80-88, 1982. 
[6] M. BLIDIA, M. CHELLALI, R. LOUNES and F. MAFFRAY: Characterizations of trees with unique minimum locating-dominating sets, Journal of Combinatorial Mathematics and Combinatorial Computing, Vol. 76, pp. 225-232, 2011.

[7] B. BREŠAN, M. A. HENNING and D. F. RALL: Rainbow domination in graphs, Taiwanese Journal of Mathematics, Vol. 12(1), pp. 213-225, 2008.

[8] C. CALABRO, R. IMPAGLIAZZO, V. KABANETS and R. PATURI: The complexity of Unique $k$-SAT: an isolation lemma for $k$-CNFs, Journal of Computer and System Sciences, Vol. 74, pp. 386-393, 2008.

[9] V. T. CHAKARAVARTHY, M. KAPRALOV, P. MURALI, F. PETRINI, X. QUE, Y. SABHARWALI and B. SCHIEBER: Subgraph counting: Color coding beyond trees, arXiv:1602.04478 (2018).

[10] I. CHARON, O. HUDRY and A. LOBSTEIN: Minimizing the size of an identifying or locating-dominating code in a graph is NP-hard, Theoretical Computer Science, Vol. 290, pp. 2109-2120, 2003.

[11] C. CHEN, C. LU and Z. MIAO: Identifying codes and locatingdominating sets on paths and cycles, Discrete Applied Mathematics, Vol. 159(15), pp. 1540-1547, 2011.

[12] G. COHEN, I. HONKALA, A. LOBSTEIN and G. ZÉMOR: On identifying codes, Proceedings of DIMACS Workshop on Codes and Association Schemes '99, Piscataway, USA, Vol. 56, pp. 97-109, 2001.

[13] C. J. COLBOURN, P. J. SLATER and L. K. STEWART: Locating dominating sets in series parallel networks, Congressus Numerantium, Vol. 56, pp. 135-162, 1987.

[14] S. A. COOK: The complexity of theorem-proving procedures, Proceedings of 3rd Annual ACM Symposium on Theory of Computing, pp. 151-158, 1971.

[15] M. FISCHERMANN: Block graphs with unique minimum dominating sets, Discrete Mathematics, Vol. 240, pp. 247-251, 2001.

[16] M. R. GAREY and D. S. JOHNSON: Computers and Intractability, a Guide to the Theory of NP-Completeness, New York: Freeman, 1979.

[17] G. GUNTHER, B. HARTNELL, L. R. MARKUS and D. RALL: Graphs with unique minimum dominating sets, Congressus Numerantium, Vol. 101, pp. 55-63, 1994.

[18] T. W. HAYNES, S. T. HEDETNIEMI and P. J. SLATER: Fundamentals of Domination in Graphs, New York: Marcel Dekker, 1998. 
[19] O. HUDRY and A. LOBSTEIN: More results on the complexity of domination problems in graphs, International Journal of Information and Coding Theory, Vol. 4, pp. 129-144, 2017.

[20] O. HUDRY and A. LOBSTEIN: Unique (optimal) solutions: complexity results for identifying and locating-dominating codes, Theoretical Computer Science, Vol. 767, pp. 83-102, 2019.

[21] O. HUDRY and A. LOBSTEIN: Complexity of unique (optimal) solutions in graphs: Vertex Cover and Domination, Journal of Combinatorial Mathematics and Combinatorial Computing, Vol. 110, pp. 217-240, 2019 .

[22] O. HUDRY and A. LOBSTEIN: Some complexity considerations on the uniqueness of solutions for satisfiability and colouring problems, submitted.

[23] D. S. JOHNSON: A catalog of complexity classes, in: Handbook of Theoretical Computer Science, Vol. A: Algorithms and Complexity, van Leeuwen, Ed., Chapter 2, Elsevier, 1990.

[24] A. LOBSTEIN: A bibliography on watching systems, identifying, locating-dominating and discriminating codes in graphs, https://www.lri.fr/ ${ }^{\sim}$ lobstein/debutBIBidetlocdom.pdf

[25] C. H. PAPADIMITRIOU: On the complexity of unique solutions, Journal of the Association for Computing Machinery, Vol. 31, pp. 392-400, 1984.

[26] C. H. PAPADIMITRIOU: Computational Complexity, Reading: Addison-Wesley, 1994.

[27] C. H. PAPADIMITRIOU and M. YANNAKAKIS: The complexity of facets (and some facets of complexity), Journal of Computer and System Sciences, Vol. 28, pp. 244-259, 1984.

[28] https://complexityzoo.uwaterloo.ca/Complexity_Zoo 